\title{
Development and Validation of a Nine-Redox-Related Long Noncoding RNA Signature in Renal Clear Cell Carcinoma
}

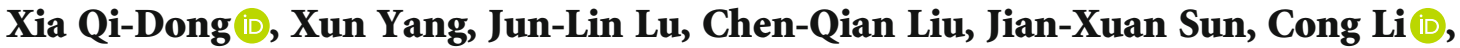 \\ and Shao-Gang Wang
}

Department and Institute of Urology, Tongji Hospital, Tongji Medical College, Huazhong University of Science and Technology, No. 1095 Jiefang Avenue, 430030 Wuhan, China

Correspondence should be addressed to Cong Li; licongtjm@163.com

Received 16 October 2020; Revised 24 November 2020; Accepted 11 December 2020; Published 28 December 2020

Academic Editor: Bin Duan

Copyright (C) 2020 Xia Qi-Dong et al. This is an open access article distributed under the Creative Commons Attribution License, which permits unrestricted use, distribution, and reproduction in any medium, provided the original work is properly cited.

\begin{abstract}
Background. Redox plays an essential role in the pathogeneses and progression of tumors, which could be regulated by long noncoding RNA (lncRNA). We aimed to develop and verify a novel redox-related lncRNA-based prognostic signature for clear cell renal cell carcinoma (ccRCC). Materials and Methods. A total of 530 ccRCC patients from The Cancer Genome Atlas (TCGA) were included in this study. All the samples were randomly split into training and test group at a $1: 1$ ratio. Then, we screened differentially expressed redox-related lncRNAs and constructed a novel prognostic signature from the training group using the least absolute shrinkage and selection operation (LASSO) and COX regression. Next, to verify the accuracy of the signature, we conducted risk and survival analysis, as well as the construction of ROC curve, nomogram, and calibration curves in the training group, test group, and all samples. Finally, the redox gene-redox-related lncRNA interaction network was constructed, and gene set enrichment analysis (GSEA) was performed to investigate the status of redox-related functions between high/low-risk groups. Results. A nine-redox-related lncRNA signature consisted of AC025580.3, COLCA1, AC027601.2, DLEU2, AC004918.3, AP006621.2, AL031670.1, SPINT1-AS1, and LAMA5-AS1 was significantly associated with overall survival in ccRCC patients. The signature proved efficient, and thus, a nomogram was successfully assembled. In addition, the GSEA results demonstrated that two major redox-related functions were enhanced in the high-risk group ccRCC patients. Conclusions. Our findings robustly demonstrate that the nine-redox-related lncRNA signature could serve as an efficient prognostic indicator for ccRCC.
\end{abstract}

\section{Introduction}

Renal cell carcinoma (RCC) is the most common malignant tumour in the kidney, accounting for nearly $90 \%$ of all kidney cancers [1]. Approximately 350,000 new cases of RCC were diagnosed worldwide per year, which caused more than 15,000 deaths per year in the USA and more than 140,000 deaths per year worldwide $[2,3]$. RCC can have several histologic subtypes; clear cell renal cell carcinoma (ccRCC) is the most common RCC subtype in adults and accounts for approximately $70 \%$ of all RCC cases [4]. The prognosis of ccRCC varies widely. For patients with early and localized disease, the cure rate is high with a 5-year survival of more than $90 \%$. However, 5-year survival was only $12 \%$ for patients with distant metastatic disease [5], which caused most deaths in ccRCC patients. In addition to the most basic surgical resection, many emerging therapies for the treatment of metastatic ccRCC have been proposed with improved knowledge of disease biology. Cabozantinib, an antiangiogenic agent that targets the VEGF pathway, was approved as a first-line therapy for patients with advanced ccRCC [6]. Immunotherapy, such as programmed cell death 1 (PD-1) and cell death-ligand 1 (PD-L1) blockers, has also been developed in ccRCC $[7,8]$. However, each of these treatments still has some limitations. Hence, it is urgent to improve the survival of patients with ccRCC. Whereas TNM (Tumor, Node, Metastasis) staging system has been the most commonly prognostic predictive system for ccRCC patients, it does not effectively predict the aggressiveness of the ccRCC [9]. Although there are several common 
prognosis factors such as tumor stage, grade, and size, these factors also do not provide accurate predictions due to their molecular and genetic heterogeneity were ignored. Identifying potential valuable molecular biomarkers would enhance the prognostic value of the developed tools.

Redox homeostasis system regulates many biological processes, including cell signaling, proliferation, and differentiation by modulating intracellular antioxidant and redox signaling (ARS). Imbalances among oxidation and antioxidation can lead to oxidative stress and damage to cell functions, contributing to a variety of diseases $[10,11]$. During the last decades, extensive research has revealed that disruption of the reduction-oxidation signaling can mediate cancer initiation and development by leading to molecular damage [12, 13]. Recent studies showed the imbalance of the redox homeostasis system is closely related to the RCC occurrence and progression $[14,15]$. Hence, it is vital to discover potential valuable redox-related biomarkers to improve the prognostic prediction of patients with ccRCC.

In recent years, scientists have focused on molecular biomarkers in the development of a reliable prognostic biomarker in cancer [16]. The long noncoding RNA (lncRNA) is a type of noncoding RNA with transcripts of $>200$ nucleotides in length without any protein-coding capacity [17], but it plays important roles in the regulation of mRNA transcription and protein translation [18]. IncRNA modulated many important biological functions, such as cell growth and survival, genomic imprinting, chromatin modifications, and allosteric regulation of enzyme activities [19]. In tumor patients, abnormal expressions of lncRNA are frequent biological phenomena and closely associated with prognosis [20]. IncRNAs have been repeatedly suggested as wellaccessible blood-based biomarkers in numerous urogenital malignancies, including RCC [21-23]. Therefore, the redoxrelated IncRNA may be used as a potential valuable biomarker or a potential therapeutic target.

In our study, we evaluated the interaction between redox and lncRNA. A nine-redox-related lncRNA signature with potential molecular prognostic value in ccRCC was identified by using both the LASSO and Cox regression analyses. We also constructed a nomogram based on this nine-redoxrelated lncRNA signature for improving the prognostic prediction of ccRCC patients, and it will serve as a reliable prognostic predictor tool for ccRCC patients in the future.

\section{Materials and Methods}

2.1. Data Sources. We searched TCGA-GDC (https://portal .gdc.cancer.gov/) for the transcriptome profiling and clinical data. We filtered the transcriptome profiling data using the following: the primary site is the kidney, the program name is TCGA, the project is TCGA-KIRC, the disease type is adenomas and adenocarcinomas, and the data category is transcriptome profiling while workflow type is HTSeq-FPKM. On the other hand, the filter criteria for clinical data included data category and format as clinical and bcr xml, respectively. We then downloaded the cart and metadata files for the transcriptome profiling data (611 samples) and the cart files for the clinical data (537 samples). The data files were decompressed and sorted into a matrix based on PERL programming. We searched the Ensembl database (http://asia.ensembl.org/index.html) for the human gene transfer format (gtf) file to transfer the gene id and annotate genes for mRNA or lncRNA. In addition, we searched GSEA-MSigDB (https://www.gsea-msigdb.org/ gsea/msigdb) for the redox-related gene set by searching "redox" as keywords, and we download two redox-related gene sets as "GO_CELL_REDOX_HOMEOSTASIS" and “GO_RESPONSE_TO_REDOX_STATE.”

\subsection{Differentially Expressed Redox-Related IncRNAs} (DERRlncRNAs). Having annotated the genes for mRNA or lncRNA, we extracted the expression of lncRNA and then used the "limma" package for the entire lncRNA data to identify the differentially expressed lncRNAs (DElncRNAs) with $|\log \mathrm{FC}|>1$ and FDR $<0.05$ between tumor and normal samples. Meanwhile, we extracted the expression of redox-related gene sets then identified redox-related lncRNAs by using the Pearson Correlation Test with $\mid$ Cor $\mid>0.5$ and p.adj $<0.001$ between lncRNAs and expression of redox-related gene sets in tumor tissue. Finally, we took an intersection of DElncRNAs and redox-related lncRNAs to screen differentially expressed redox-related lncRNAs (DERRlncRNAs).

2.3. Random Grouping and Signature Construction. We merge the expression of DERRIncRNAs with their clinical survival data; then, all the samples were randomly split into the training and test groups at a 1:1 ratio. Following this, we performed univariate Cox regression of DERRlncRNAs in the training group to identify prognosis-related DERRlncRNAs with the filter criterion set at a significance of $p<0.05$. Also, to avoid overfitting, we applied LASSO regression to screen appropriate variables from the prognosis-related DERRlncRNAs. Finally, a survival-predicting model was constructed by a multivariate Cox proportional hazard model. Importantly, a risk score formula was created based on the signature: Risk score $=\sum_{i=1}^{N}(\operatorname{Exp}(i) \bullet \operatorname{coe}(i)) . N$ is the number of redox-related IncRNA in the multivariate COX regression, $\operatorname{Exp}(\mathrm{i})$ is the expression value of $\operatorname{lncRNA}$, and Coe(i) is the estimated regression coefficient of lncRNA in the multivariate Cox regression analysis. Then, the samples in both the training group and test group obtained a risk score calculated by the formula, and we set the medium value of the risk score in the training group as filter criteria that the higher risk score is high risk and the lower risk score is low risk.

2.4. Validation of the Survival-Predicting Model. According to the risk level judged by the risk score, we performed the Kaplan-Meier method survival analysis to test the survivalpredicting availability of the signature and plot the survival curve for the samples in the training group, test group, and all group. Then, we merged the clinical data which contained age, gender, stage, and grade with the risk score of patients and rechecked to delete samples lacking accurate clinical data. Following this, we plotted the multivariate ROC curves to verify and compare the efficacy of the developed signature with the other clinical prognostic factors; the area under the curve (AUC) for multiple factors which contained age, 
TABLE 1: Clinical characteristics of the KIRC patients.

\begin{tabular}{|c|c|c|c|c|}
\hline & Overall & Test & Train & $p$ \\
\hline$n$ & 530 & 264 & 266 & \\
\hline Age (mean (SD)) & $60.56(12.14)$ & $60.48(11.76)$ & $60.65(12.52)$ & 0.875 \\
\hline Gender $=$ female/male $(\%)$ & $186 / 344(35.1 / 64.9)$ & $82 / 182(31.1 / 68.9)$ & $104 / 162(39.1 / 60.9)$ & 0.065 \\
\hline Grade (\%) & & & & 0.432 \\
\hline G1 & $14(2.6)$ & $5(1.9)$ & $9(3.4)$ & \\
\hline G2 & $227(42.8)$ & $108(40.9)$ & $119(44.7)$ & \\
\hline G3 & $206(38.9)$ & $110(41.7)$ & $96(36.1)$ & \\
\hline G4 & $75(14.2)$ & $36(13.6)$ & $39(14.7)$ & \\
\hline GX & $5(0.9)$ & $4(1.5)$ & $1(0.4)$ & \\
\hline Unknown & $3(0.6)$ & $1(0.4)$ & $2(0.8)$ & \\
\hline Stage (\%) & & & & 0.169 \\
\hline Stage I & $265(50.0)$ & $125(47.3)$ & $140(52.6)$ & \\
\hline Stage II & $57(10.8)$ & $26(9.8)$ & $31(11.7)$ & \\
\hline Stage III & $123(23.2)$ & $68(25.8)$ & $55(20.7)$ & \\
\hline Stage IV & $82(15.5)$ & $45(17.0)$ & $37(13.9)$ & \\
\hline Unknown & $3(0.6)$ & $0(0.0)$ & $3(1.1)$ & \\
\hline $\mathrm{T}(\%)$ & & & & 0.414 \\
\hline $\mathrm{T} 1$ & $21(4.0)$ & $10(3.8)$ & $11(4.1)$ & \\
\hline T1a & $140(26.4)$ & $68(25.8)$ & $72(27.1)$ & \\
\hline $\mathrm{T} 1 \mathrm{~b}$ & $110(20.8)$ & $49(18.6)$ & $61(22.9)$ & \\
\hline $\mathrm{T} 2$ & $55(10.4)$ & $27(10.2)$ & $28(10.5)$ & \\
\hline $\mathrm{T} 2 \mathrm{a}$ & $10(1.9)$ & $3(1.1)$ & $7(2.6)$ & \\
\hline $\mathrm{T} 2 \mathrm{~b}$ & $4(0.8)$ & $1(0.4)$ & $3(1.1)$ & \\
\hline $\mathrm{T} 3$ & $5(0.9)$ & $2(0.8)$ & $3(1.1)$ & \\
\hline $\mathrm{T} 3 \mathrm{a}$ & $120(22.6)$ & $69(26.1)$ & $51(19.2)$ & \\
\hline $\mathrm{T} 3 \mathrm{~b}$ & $52(9.8)$ & $30(11.4)$ & $22(8.3)$ & \\
\hline $\mathrm{T} 3 \mathrm{c}$ & $2(0.4)$ & $0(0.0)$ & $2(0.8)$ & \\
\hline $\mathrm{T} 4$ & $11(2.1)$ & $5(1.9)$ & $6(2.3)$ & \\
\hline M (\%) & & & & 0.283 \\
\hline M0 & $420(79.2)$ & $209(79.2)$ & $211(79.3)$ & \\
\hline M1 & $78(14.7)$ & $43(16.3)$ & $35(13.2)$ & \\
\hline MX & $30(5.7)$ & $12(4.5)$ & $18(6.8)$ & \\
\hline Unknown & $2(0.4)$ & $0(0.0)$ & $2(0.8)$ & \\
\hline $\mathrm{N}(\%)$ & & & & 0.68 \\
\hline No & $239(45.1)$ & $124(47.0)$ & $115(43.2)$ & \\
\hline N1 & $16(3.0)$ & $8(3.0)$ & $8(3.0)$ & \\
\hline $\mathrm{NX}$ & $275(51.9)$ & $132(50.0)$ & $143(53.8)$ & \\
\hline Risk = high/low (\%) & $271 / 259(51.1 / 48.9)$ & $138 / 126(52.3 / 47.7)$ & $133 / 133(50.0 / 50.0)$ & 0.663 \\
\hline
\end{tabular}

gender, stage, grade, and risk scores was calculated and compared with each other in the training group, test group, and all samples.

2.5. Construction and Validation of the Risk Score-Based Nomogram. To provide clinicians with a quantitative rather than qualitative approach for predicting survival, we assembled a nomogram according to the risk score and clinicopathologic characteristics from the samples in the training group, then performed internal cross-validation, and input test group and all samples as two external validation set to per- form an external validation. All the calibration curve for 1 year, 3 years, and 5 years were plotted.

2.6. Gene Set Enrichment Analysis (GSEA) and Clinical Correlation. Though we had tested the survival-predicting availability of the signature, how the redox-related functions worked was still unknown; thus, we divided the transcriptome file for all samples into the high-risk group and low-risk group according to the medium value of risk score in the training group and then exported the data as "cls" and "gct" format files, which were then imported into GSEA 


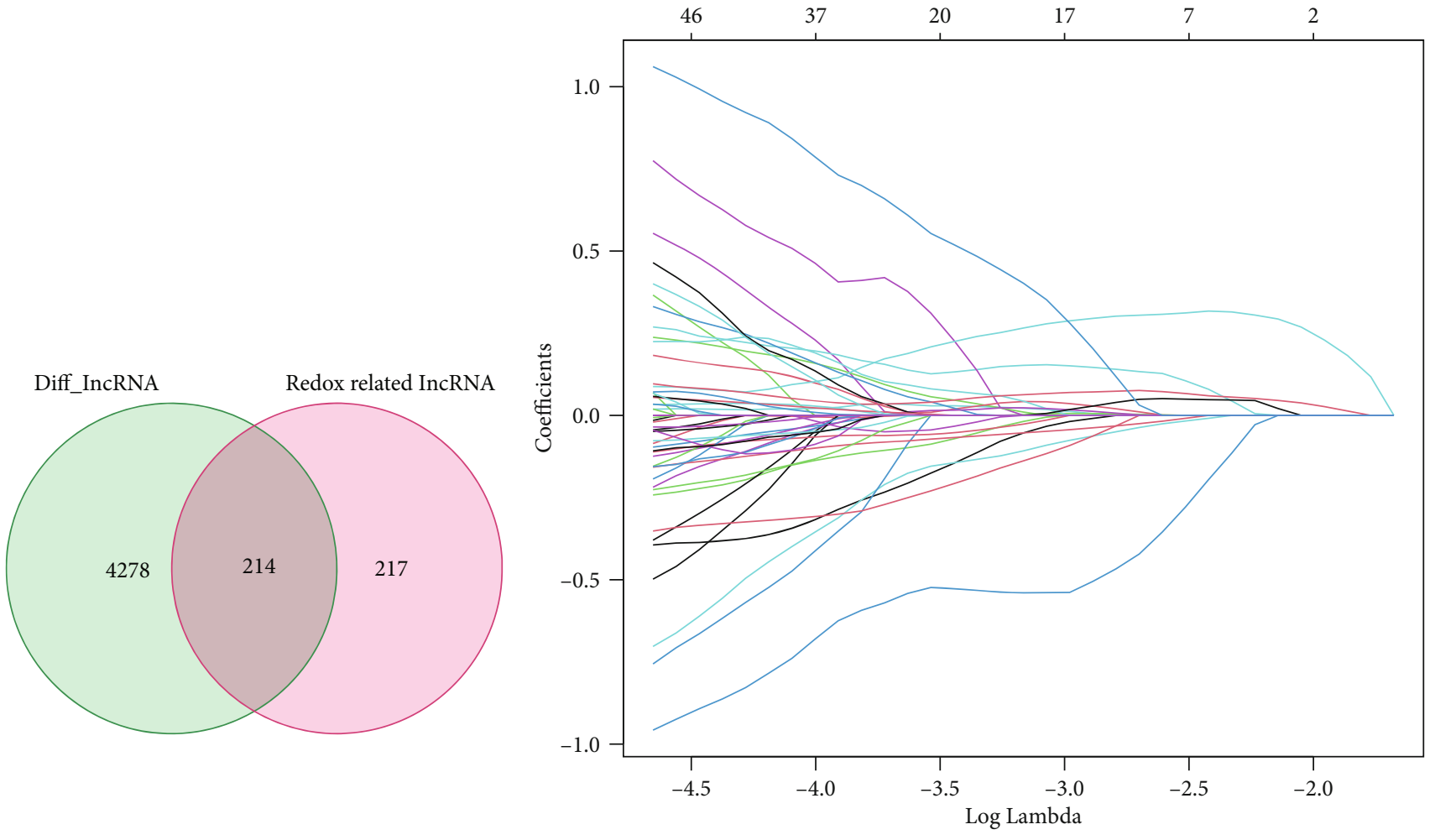

(a)

(b)

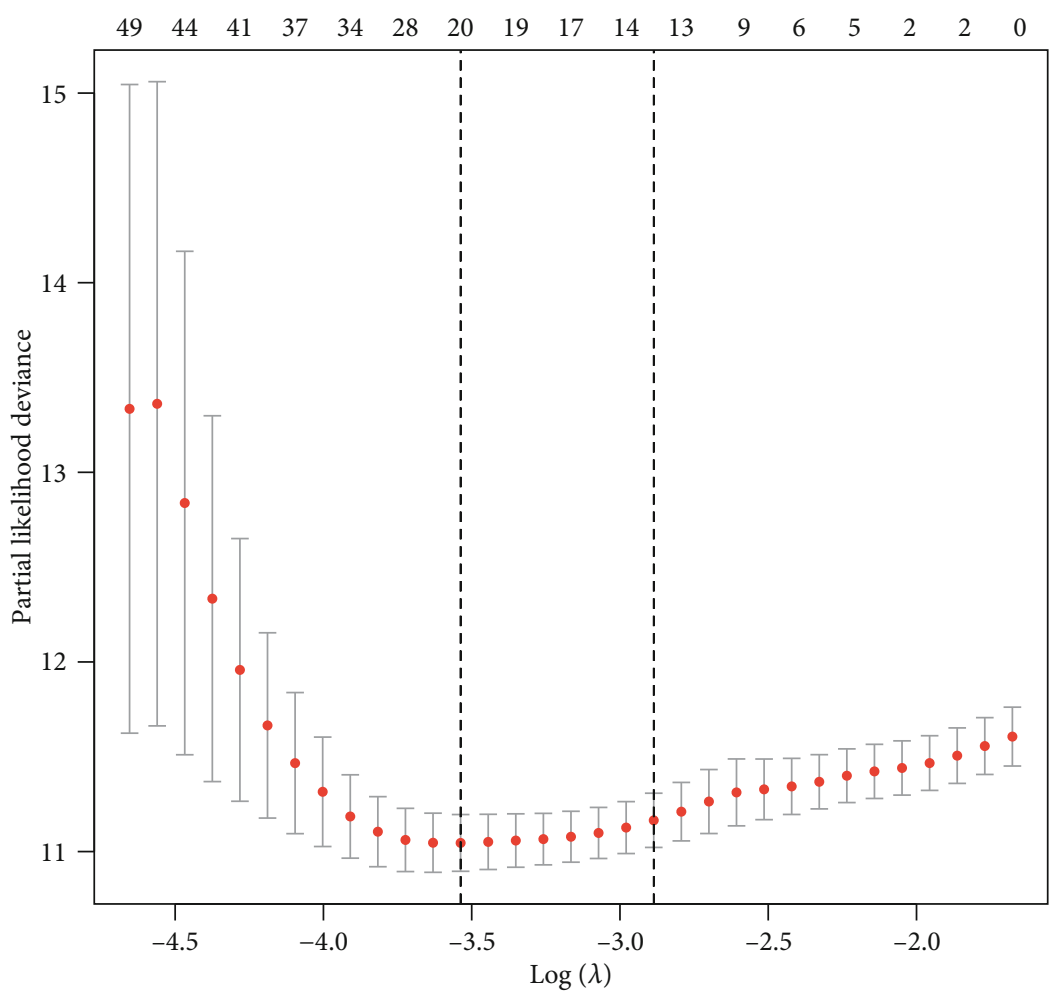

(c)

Figure 1: Continued. 


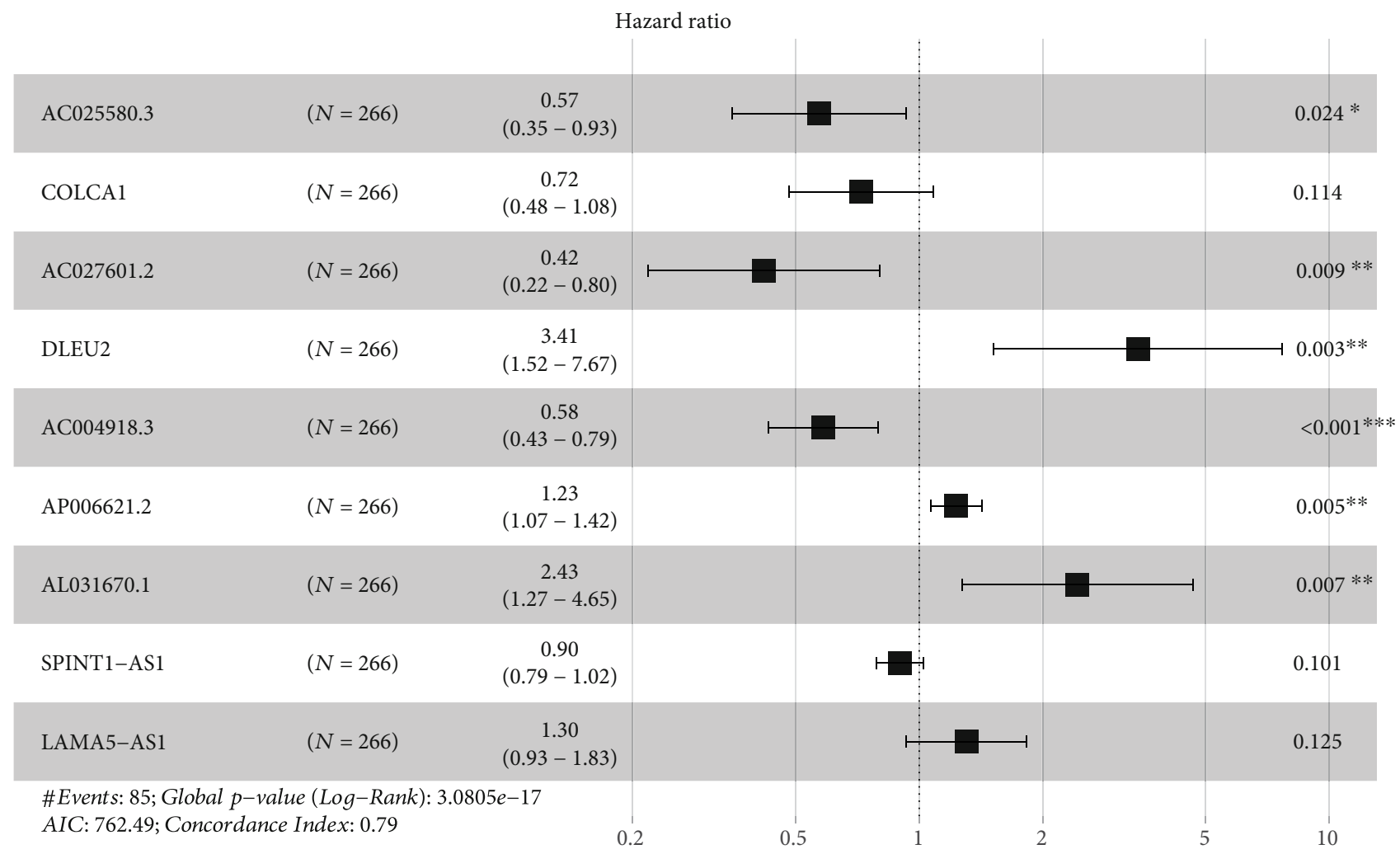

(d)

FIGURE 1: Development of the nine redox-related lncRNAs signature. (a) Screening of differentially expressed redox-related lncRNAs. (b) Variables going to zero as we increase the penalty (lambda) in the objective function of the LASSO. (c) 10-fold cross-validation for tuning parameter selection in the LASSO model, $-4<$ lambda.min $<-3.5$, and there were 20 variables (lncRNAs) left. (d) Results of the multivariate Cox proportional hazard model based on the 20 variables; nine lncRNA genes were screened to construct the signature.

TABLE 2: The detailed information of the nine redox-related lncRNAs used to construct the prognostic signature.

\begin{tabular}{llcr}
\hline Gene symbol & Ensemble ID & Gene_biotype & Coef \\
\hline AC025580.3 & ENSG00000275672 & Antisense (lncRNA) & -0.56136127 \\
COLCA1 & ENSG00000196167 & Antisense (lncRNA) & -0.326969031 \\
AC027601.2 & ENSG00000262115 & Antisense (lncRNA) & -0.873174537 \\
DLEU2 & ENSG00000231607 & Antisense (lncRNA) & 1.228067267 \\
AC004918.3 & ENSG00000270157 & Sense intronic & -0.539291321 \\
AP006621.2 & ENSG00000255142 & lincRNA & 0.207380745 \\
AL031670.1 & ENSG00000275582 & Antisense (lncRNA) & 0.889174399 \\
SPINT1-AS1 & ENSG00000261183 & Antisense (lncRNA) & -0.10982088 \\
LAMA5-AS1 & ENSG00000228812 & Antisense (lncRNA) & 0.264867342 \\
\hline
\end{tabular}

Notes: Antisense: transcripts that overlap the genomic span (i.e., exon or introns) of a protein-coding locus on the opposite strand. Sense intronic: a long noncoding transcript in introns of a coding gene that does not overlap any exons. lincRNA (long intergenic ncRNA): transcripts that are long intergenic noncoding RNA locus with a length $>200 \mathrm{bp}$. Requires lack of coding potential and may not be conserved between species.

(version 4.0.3), and conducted the analysis to explore whether the redox-related functions were significantly differentially enriched between the two groups. In addition, the correlation between risk level and clinicopathologic characteristics were tested, and the differential expression of the nine redox-related lncRNAs between the high-risk and lowrisk group was analyzed.
2.7. Coexpression Network, Correlation Plot, and Differential Expression Status. Having validated the efficacy of the nine redox-related lncRNA survival-predicting signature, we extracted the coexpression status of the redox genes and redox-related lncRNA from the primary PEARSON Correlation Test then used Cytoscape (version 3.8.0) to visualize the coexpression network. Also, the Sankey plot and correlation 


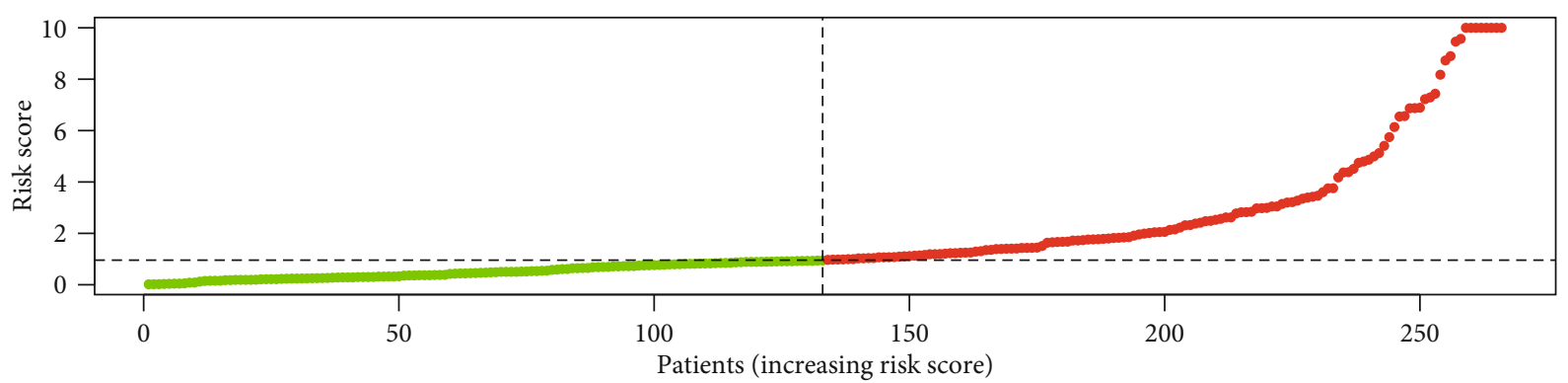

- High risk

Low risk

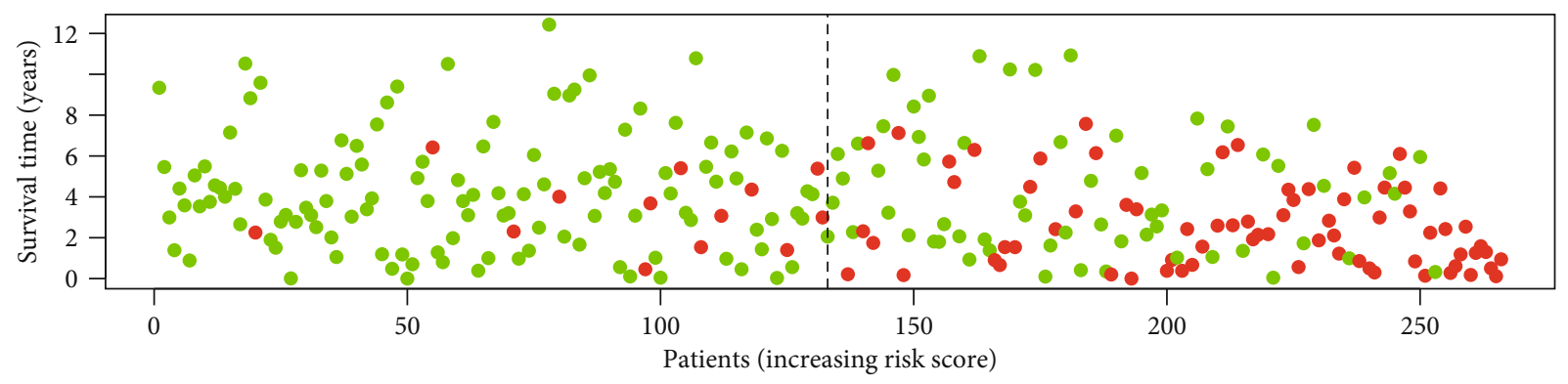

- Dead

- Alive

(a)

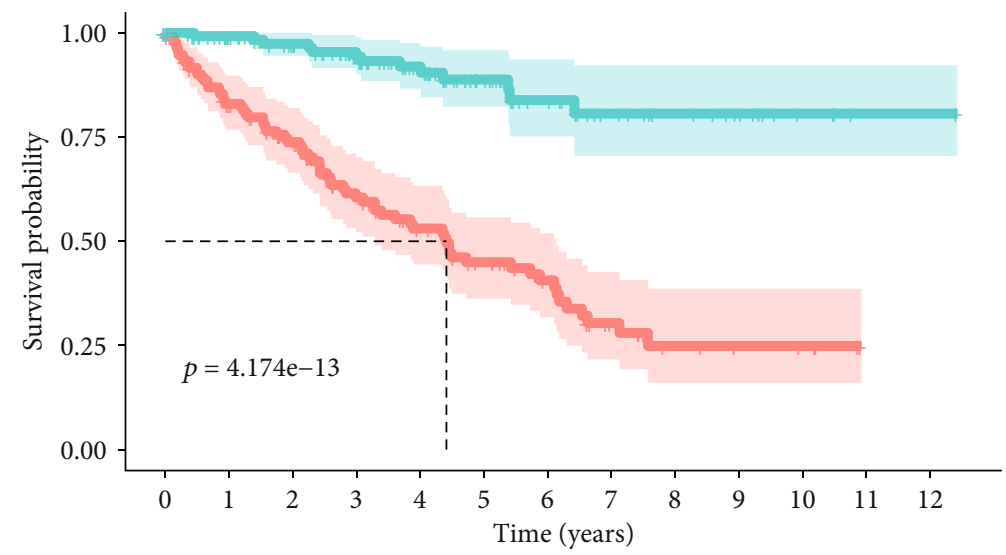

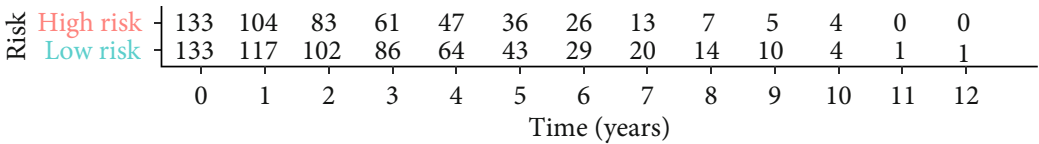

Risk

1 High risk

Low risk

(b)

Figure 2: Continued. 

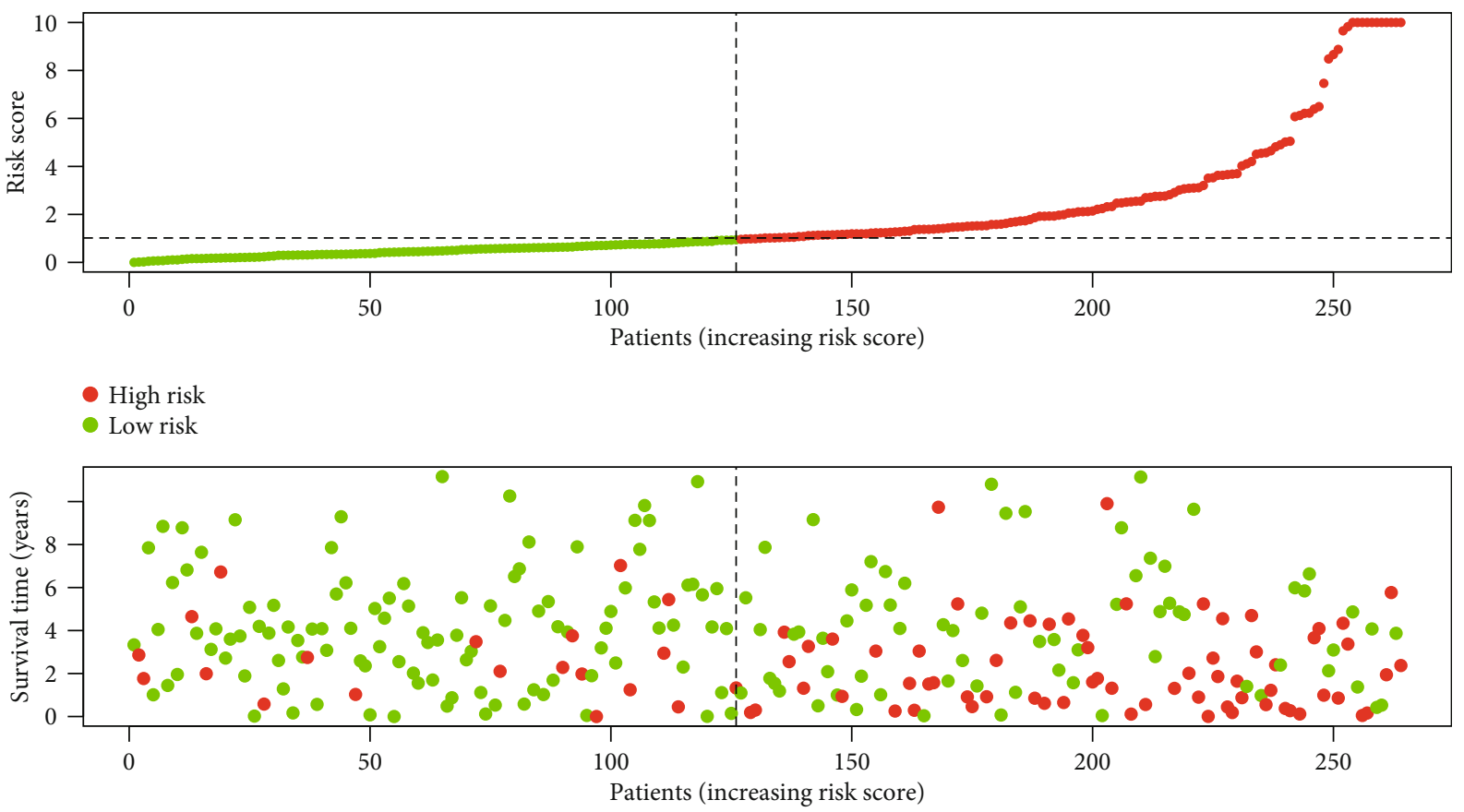

- Dead

- Alive

(c)

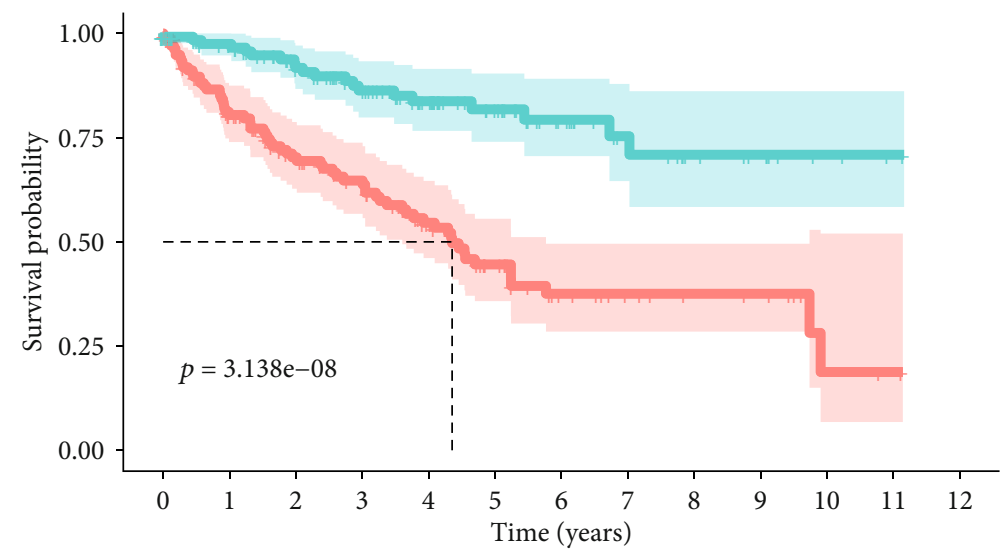

\begin{tabular}{|c|c|c|c|c|c|c|c|c|c|c|c|c|c|}
\hline \multirow{3}{*}{$\begin{array}{l}\text { He High risk } \\
\text { בै Low risk }\end{array}$} & 138 & 104 & $\begin{array}{l}79 \\
91\end{array}$ & $\begin{array}{l}67 \\
76\end{array}$ & $\begin{array}{l}48 \\
58\end{array}$ & $\begin{array}{l}30 \\
40\end{array}$ & $\begin{array}{l}17 \\
26\end{array}$ & 12 & 9 & $\begin{array}{l}8 \\
8\end{array}$ & $\begin{array}{l}2 \\
3\end{array}$ & 1 & $\begin{array}{l}0 \\
0\end{array}$ \\
\hline & 126 & 110 & & & & & 20 & 11 & 11 & & & & \\
\hline & 0 & 1 & 2 & 3 & 4 & $\begin{array}{l}5 \\
\mathrm{~T}\end{array}$ & 6 & $\begin{array}{r}7 \\
\text { s) }\end{array}$ & 8 & 9 & 10 & 11 & 12 \\
\hline
\end{tabular}

Risk

는 High risk

L. Low risk

(d)

FIgure 2: Continued. 


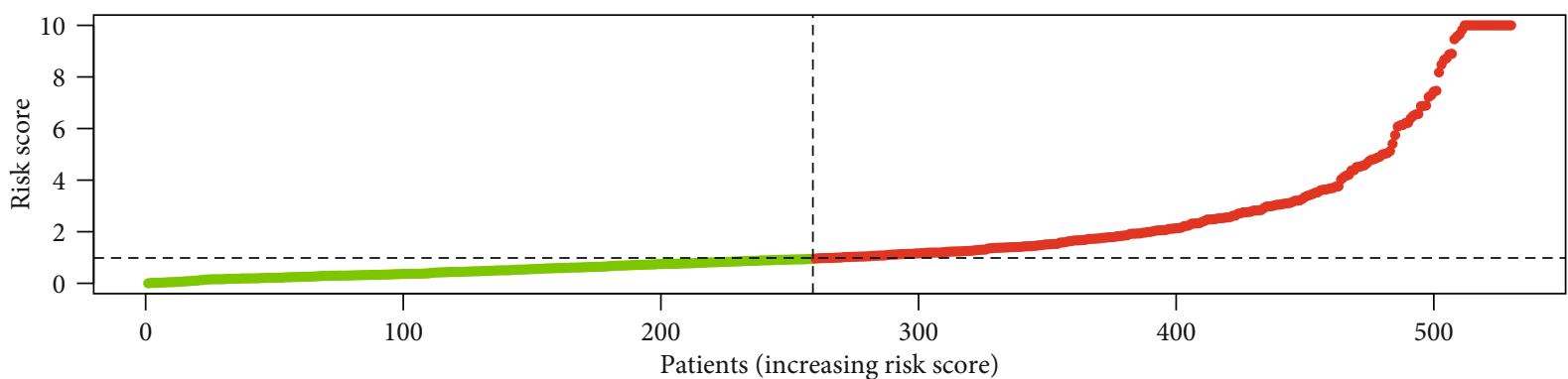

- High risk

- Low risk

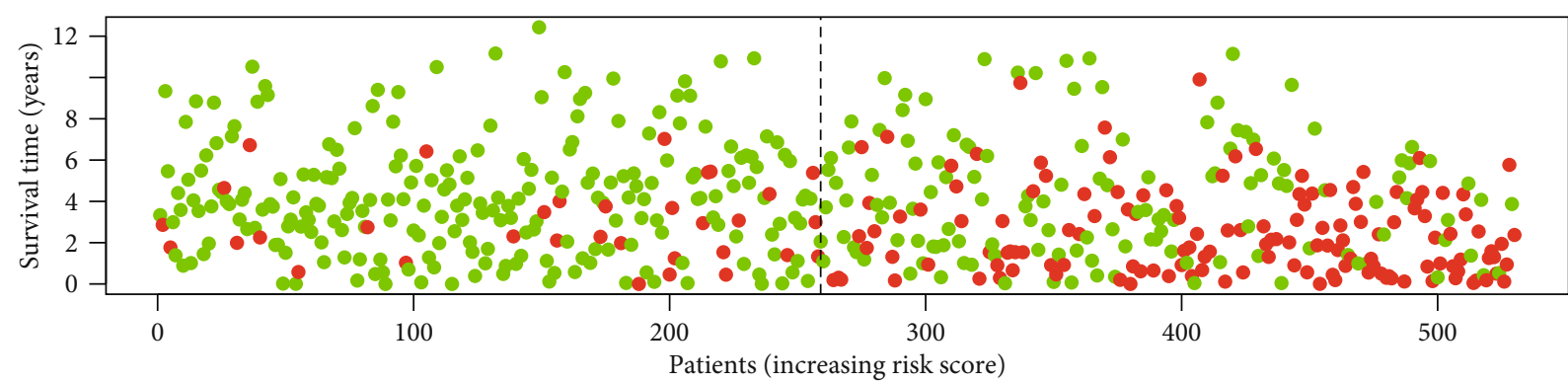

- Dead

- Alive

(e)

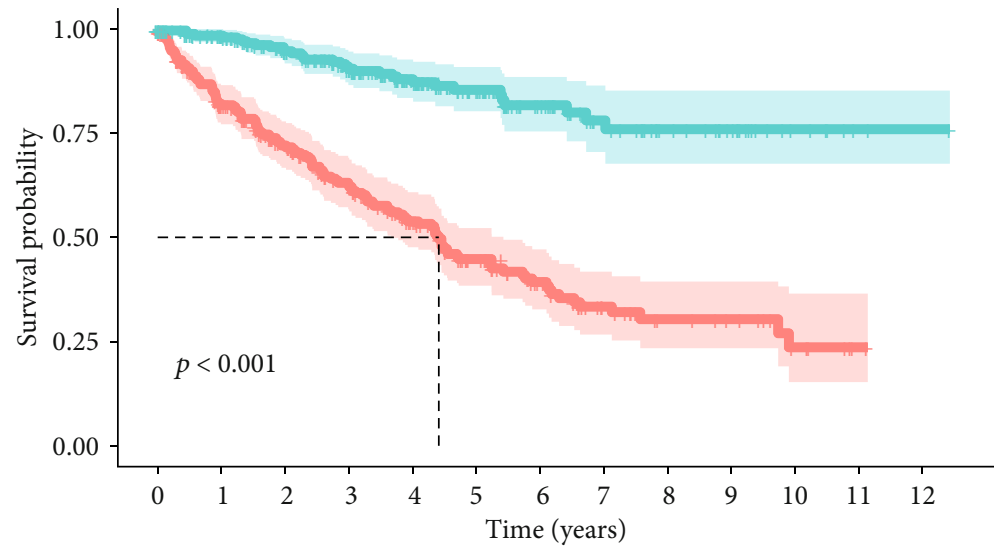

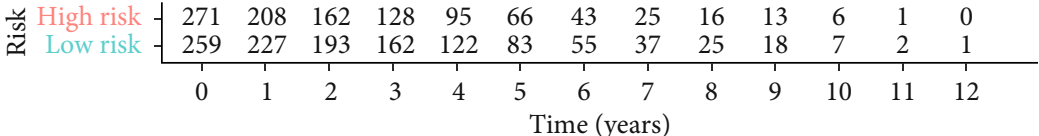

Risk

I. High risk

Low risk

(f)

FIgURE 2: Risk plot and survival curves. (a) Risk plot of the training group. (b) Survival curve of the training group. (c) Risk plot of the test group. (d) Survival curve of the test group. (e) Risk plot of all samples. (f) Survival curve of all samples. 


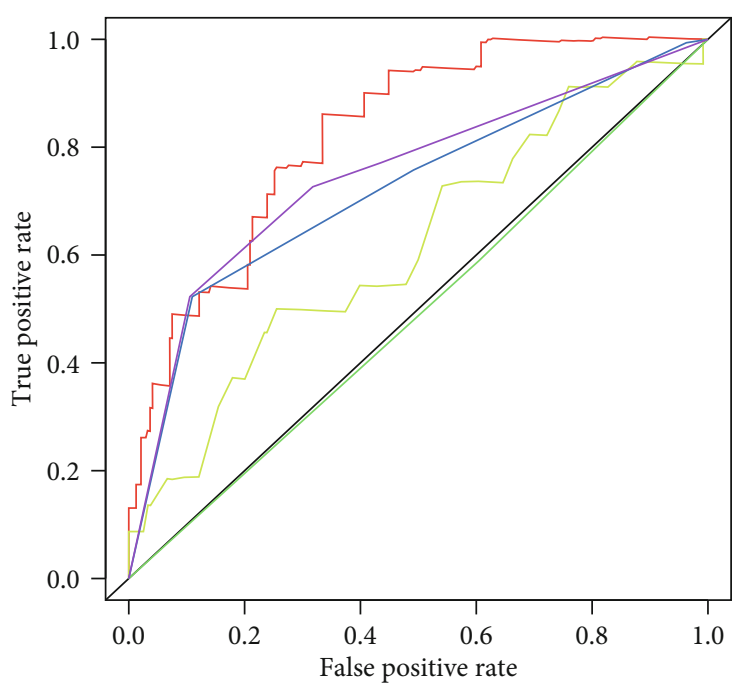

$\begin{array}{ll}\text { Risk score }(\mathrm{AUC}=0.826) & - \text { Grade }(\mathrm{AUC}=0.723) \\ \text { Age }(\mathrm{AUC}=0.618) & - \text { Stage }(\mathrm{AUC}=0.748) \\ \text { Gender }(\mathrm{AUC}=0.492) & \end{array}$

(a)

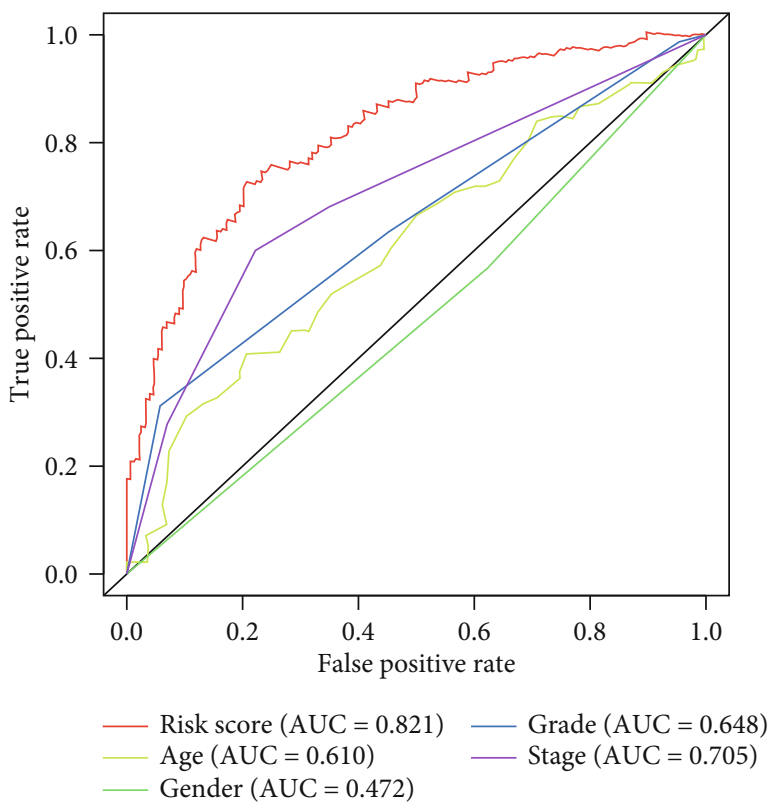

(c)

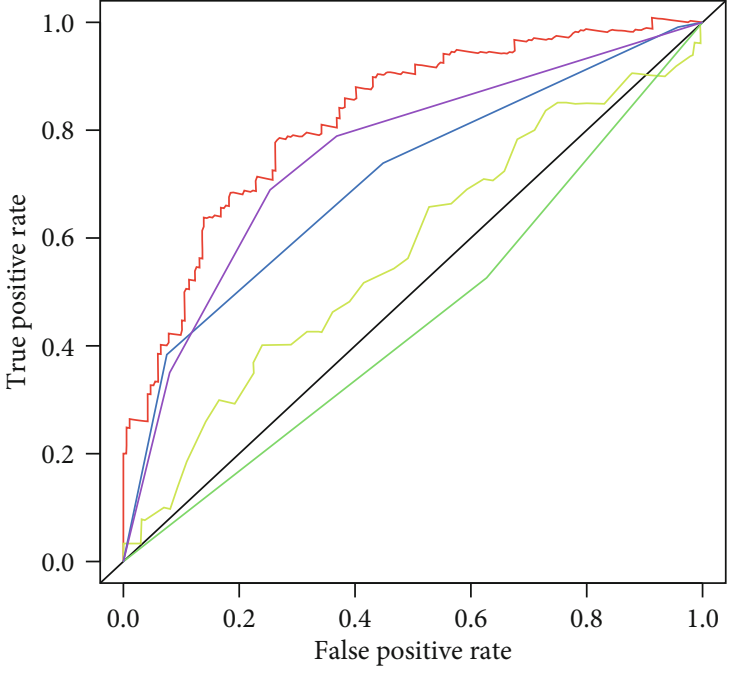

$\begin{array}{ll}\text { - Risk score }(\mathrm{AUC}=0.822) & \text { Grade }(\mathrm{AUC}=0.707) \\ \text { Age }(\mathrm{AUC}=0.573) & \text { Stage }(\mathrm{AUC}=0.754) \\ \text { Gender }(\mathrm{AUC}=0.449) & \end{array}$

(b)

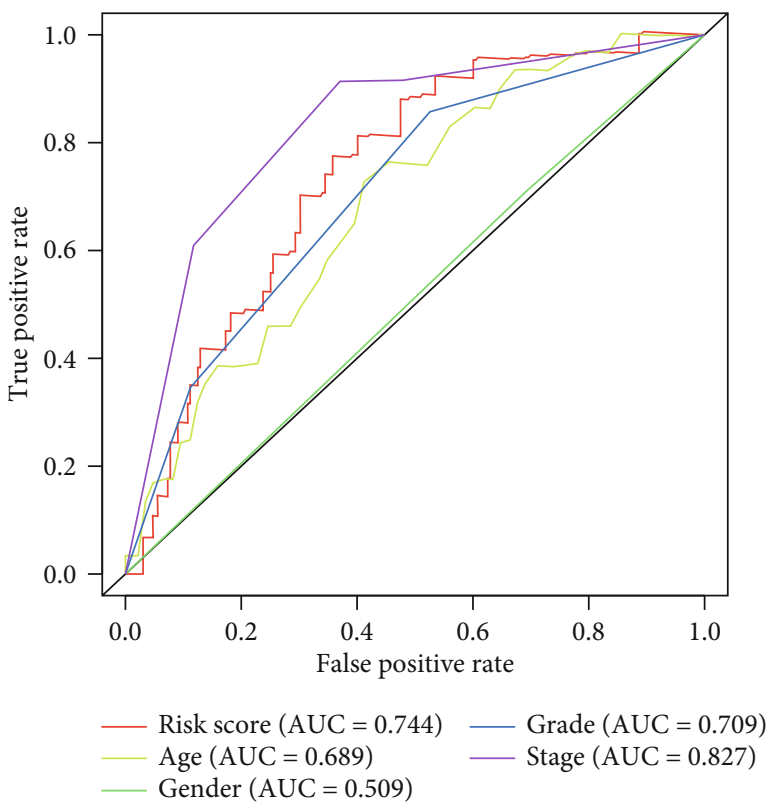

(d)

Figure 3: Continued. 


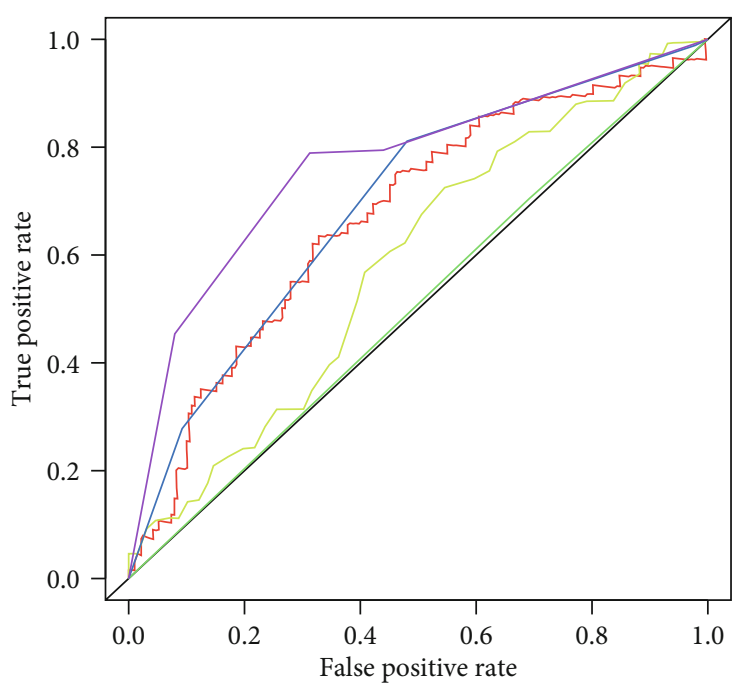

$\begin{array}{ll}\text { Risk score }(\mathrm{AUC}=0.673) & - \text { Grade }(\mathrm{AUC}=0.694) \\ \text { Age }(\mathrm{AUC}=0.586) & - \text { Stage }(\mathrm{AUC}=0.766) \\ \text { Gender }(\mathrm{AUC}=0.506) & \end{array}$

(e)

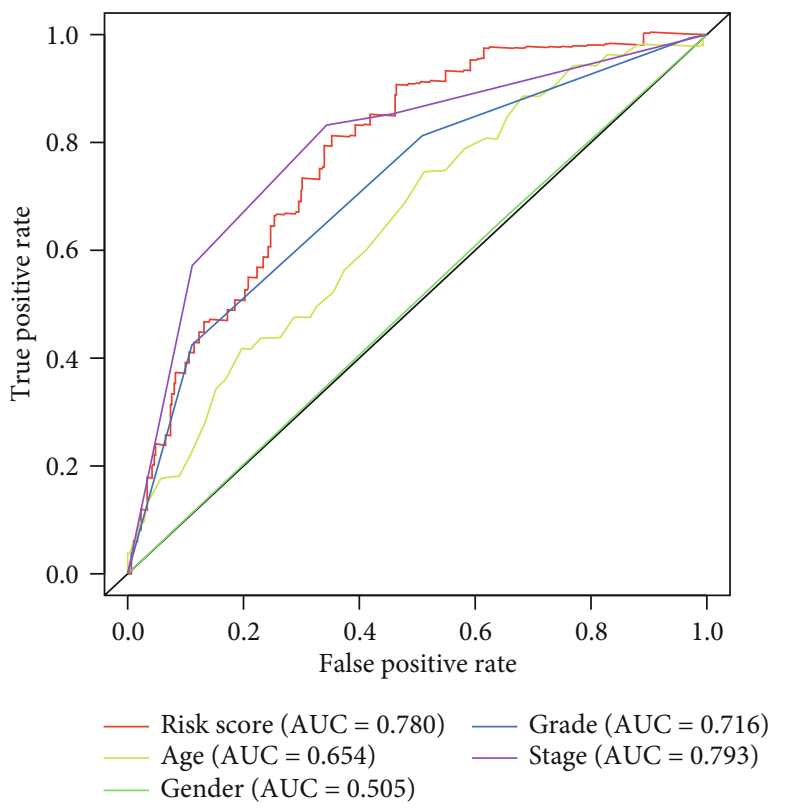

(g)
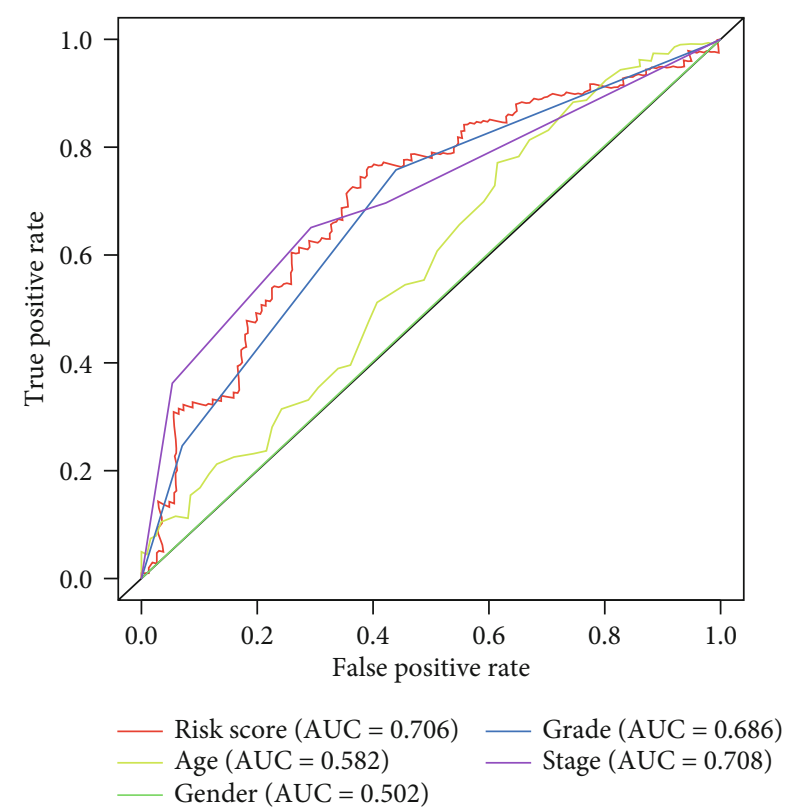

(f)

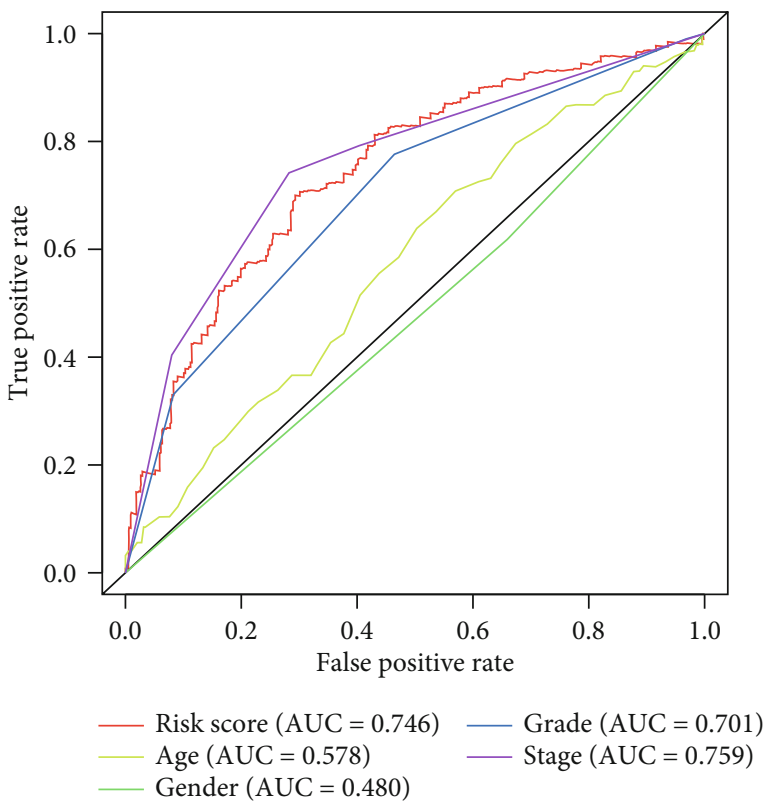

(h)

Figure 3: Continued. 


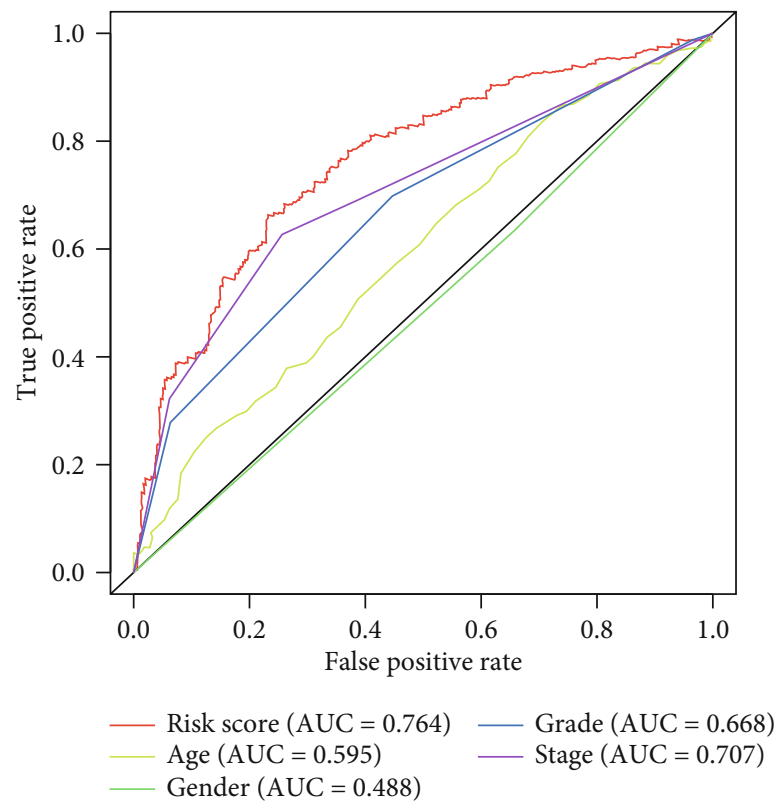

(i)

FIGURE 3: Time-dependent multivariate ROC curve. (a) One-year multivariate ROC curve in the training group. (b) Three-year multivariate ROC curve in the training group. (c) Five-year multivariate ROC curve in the training group. (d) One-year multivariate ROC curve in the test group. (e) Three-year multivariate ROC curve in the test group. (f) Five-year multivariate ROC curve in the test group. (g) One-year multivariate ROC curve in all samples. (h) Three-year multivariate ROC curve in all samples. (i) Five-year multivariate ROC curve in all samples.

circle plot were used to visualize the correlation between redox genes and nine redox-related lncRNAs. In addition, the differential expression of these nine redox-related lncRNAs between normal and tumor tissue, and between different clinicopathologic characteristics were analyzed and plotted.

2.8. Subgroup Analysis. To further explore the correlation between risk score and clinicopathological characteristics and verify the effectiveness of the prognostic signature in different clinicopathological subgroups, all samples were divided into subgroups according to age ( $>65$ or $\leq 65)$, gender (male or female), stage (stage I-II or stage III-IV), and grade (G1-2 or G3-4). Then, we compared the mean risk score between the different groups and performed survival analysis to validate the effectiveness of our prognostic signature in different subgroups.

2.9. Statistical Analysis. The data was processed using the Strawberry PERL programming language (version 5.30.2.1). All statistical analyses were performed using the $\mathrm{R}$ software (version 4.0.2). $p<0.05$ was regarded as statistically significant.

\section{Results}

3.1. Patients and Samples. There were 611 transcriptome profiles that contained 72 normal tissues and 539 tumor tissues from 530 KIRC patients, and we took the average of the tumor samples sequenced multiple times. Also, all samples were randomly split into the training and test group at a
$1: 1$ ratio, and the characteristic of the samples in the training group, test group, and all samples are shown in Table 1. Fisher's exact test was performed to compare the differences between groups. It seemed that there was no significant difference between these groups.

3.2. Differentially Expressed Redox-Related IncRNAs (DERRlncRNAs). As shown in Figure 1(a), there are a total of 4492 differentially expressed lncRNAs with $|\operatorname{logFC}|>1$ andFDR $<0.05$. And a total of 431 redox-related lncRNAs were screened with Pearson correlation coefficient $\mid$ cor $\mid>$ 0.5 and p.adjust $<0.001$. Then, we took an intersection of them and acquired 214 differentially expressed redoxrelated lncRNAs (DERRlncRNAs).

3.3. Construction of the Redox-Related IncRNA SurvivalPredicting Signature. In the training group, we performed univariate Cox regression and got 88 significant prognostic DERRlncRNAs. Then, the LASSO regression was used to avoid overfitting and screened 20 appropriate DERRIncRNAs as variates to do the following multivariate cox regression (Figures 1(b) and 1(c)). Finally, we performed multivariate Cox regression and developed a nine-redox-related lncRNA signature containing AC025580.3, COLCA1, AC027601.2, DLEU2, AC004918.3, AP006621.2, AL031670.1, SPINT1-AS1, and LAMA5-AS1 to predict the survival of KIRC patients (Figure 1(d)), and their detailed information is shown in Table 2. The risk score for each sample was then calculated based on the expression levels of these nine redox-related lncRNAs. $\quad$ Risk score $=1.23 \cdot$ DLEU2 $+0.21 \cdot A P 006621.2+$ $0.89 \bullet A L 031670.1+0.26 \bullet L A M A 5-A S 1-0.56 \bullet A C 025580.3$ 


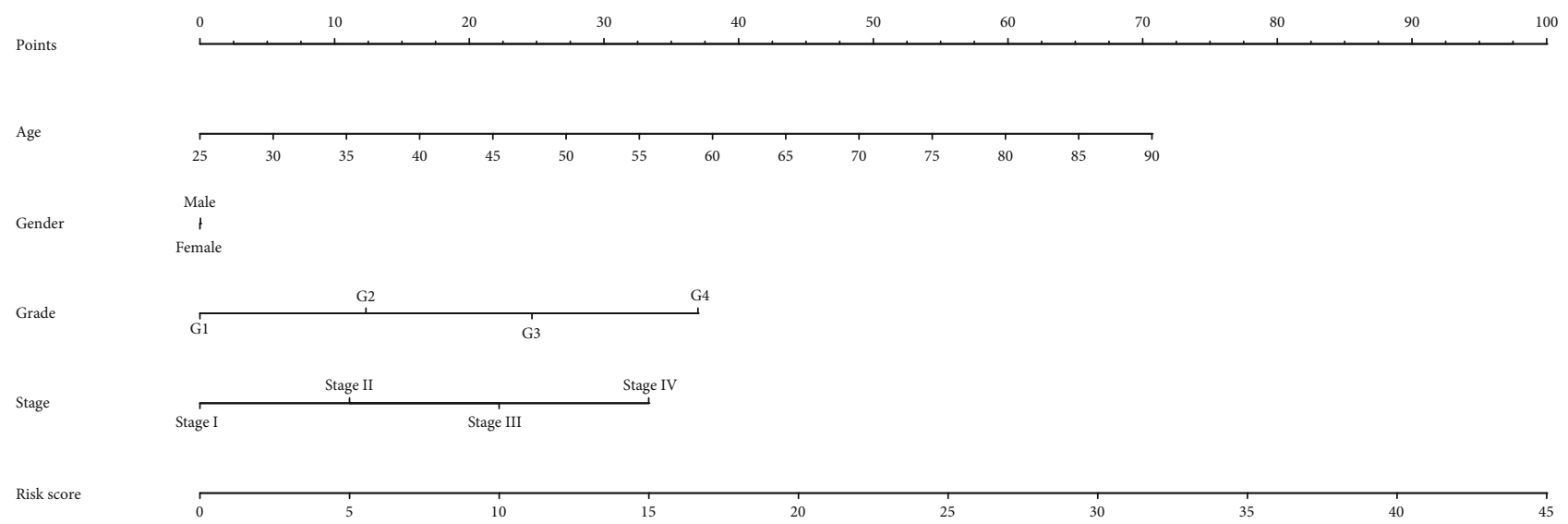

Total Points

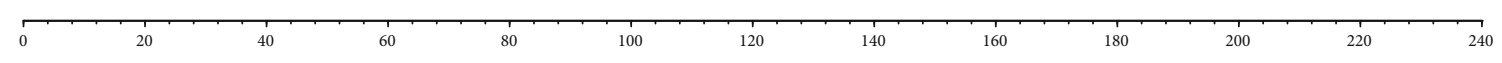

1-year survival

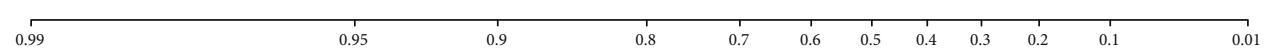

3-year survival

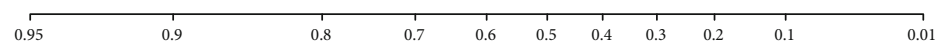

5-year survival

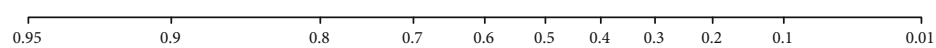

(a)

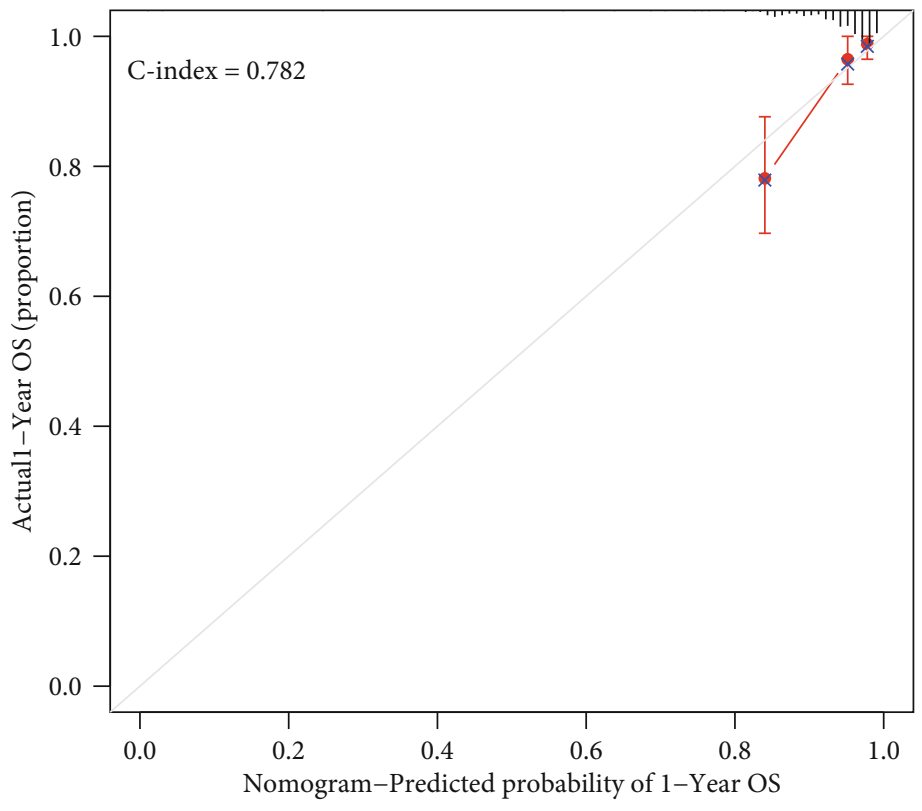

(b)

Figure 4: Continued. 


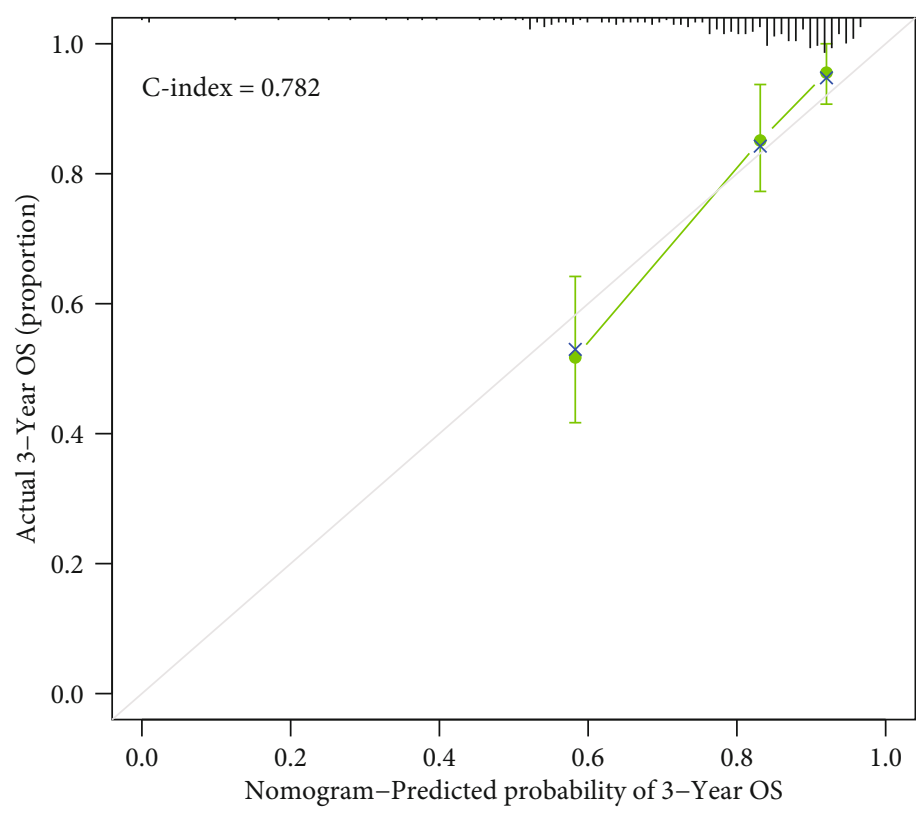

(c)

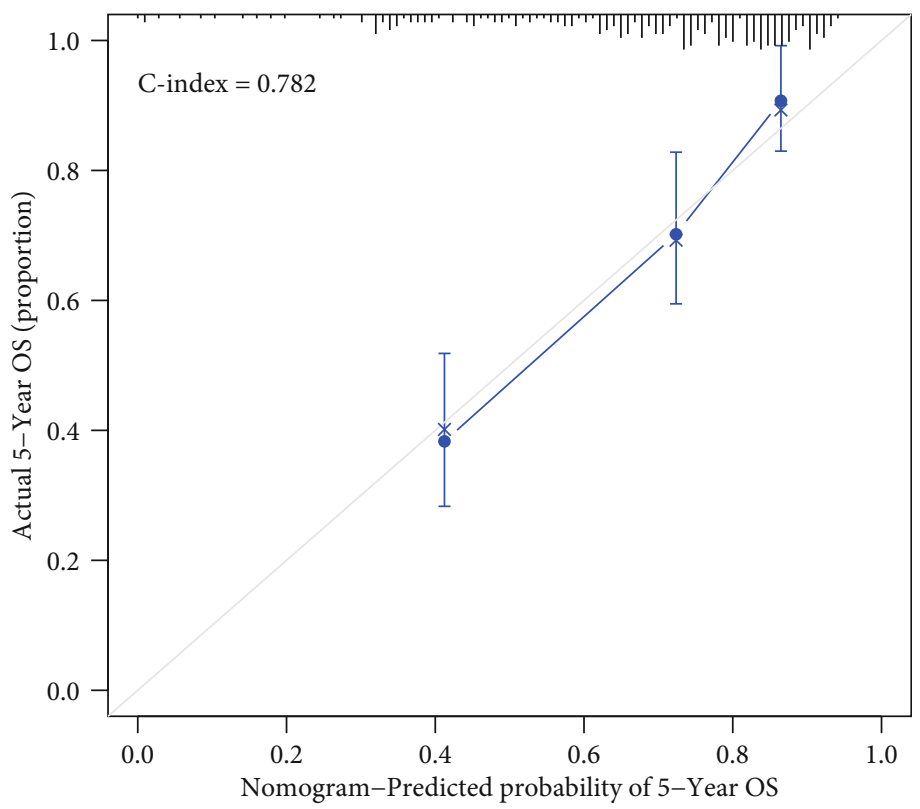

(d)

FIgURE 4: Continued. 


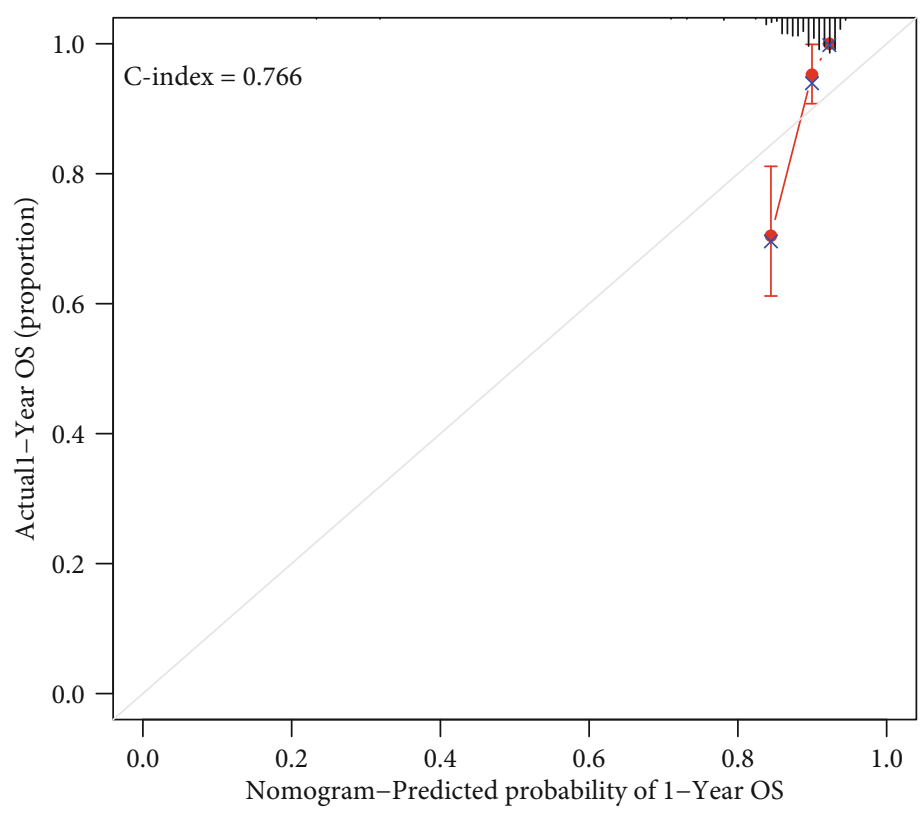

(e)

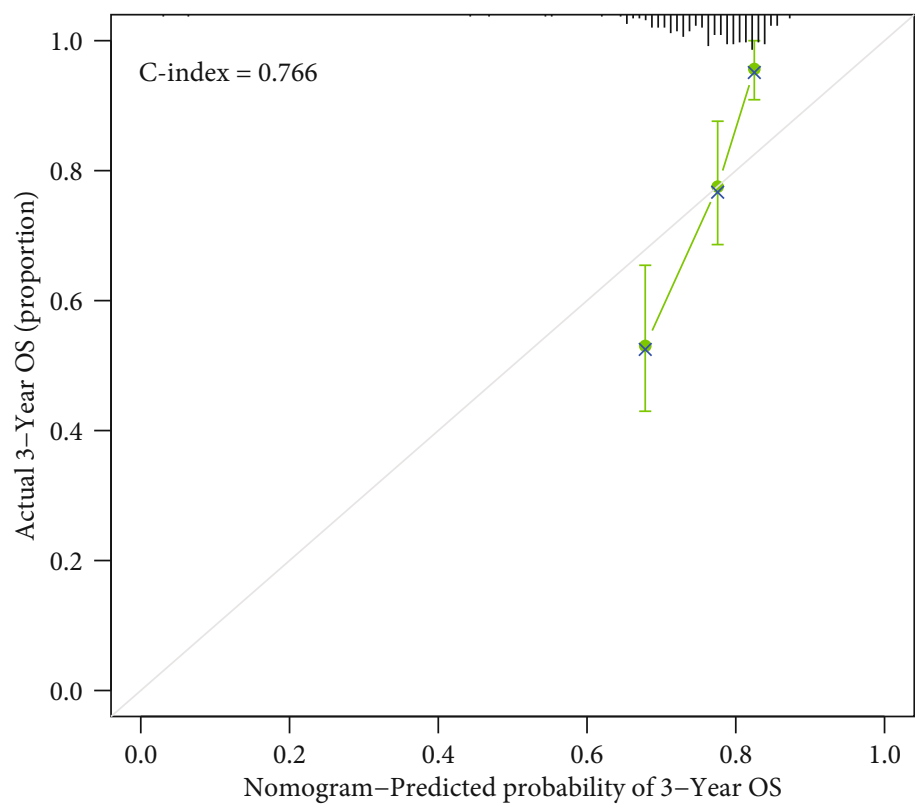

(f)

FIgURe 4: Continued. 


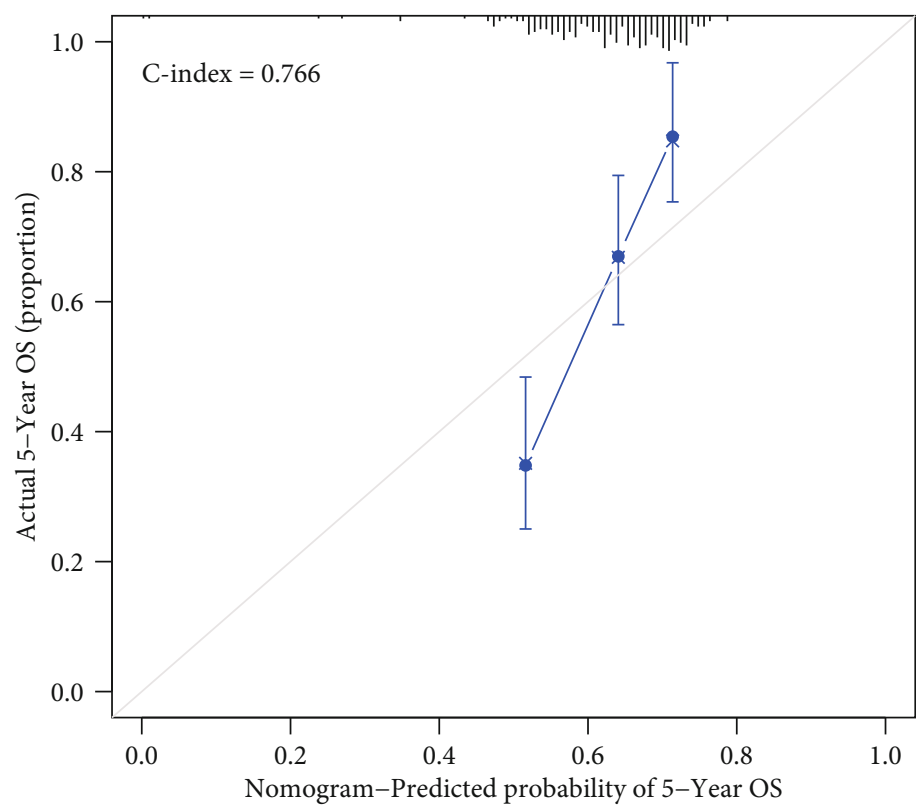

(g)

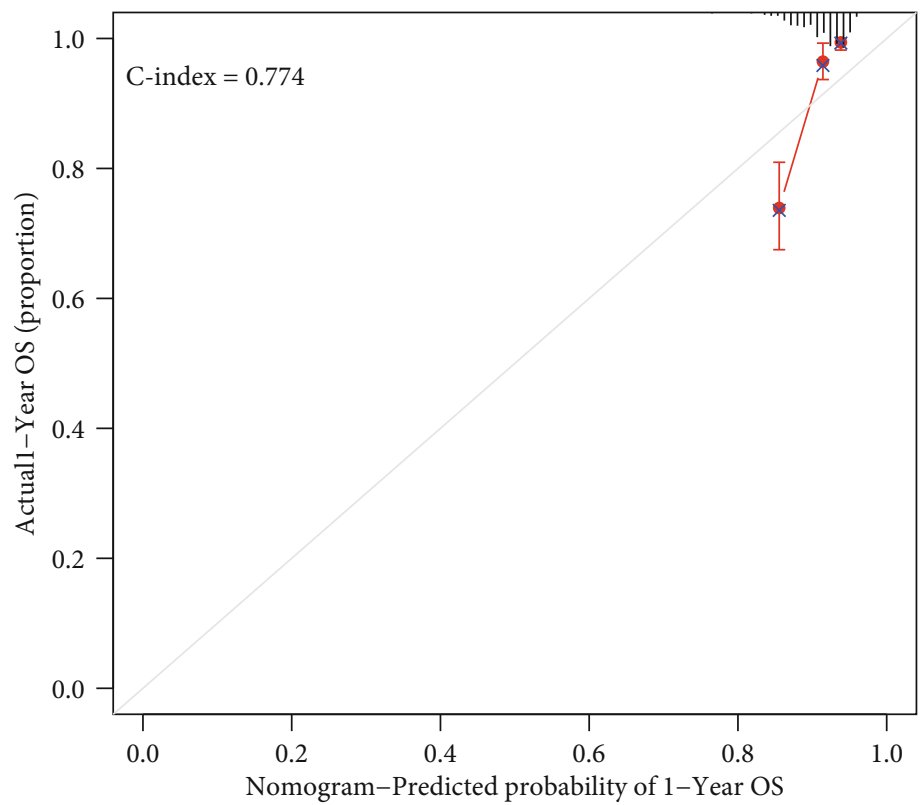

(h)

FIgURE 4: Continued. 


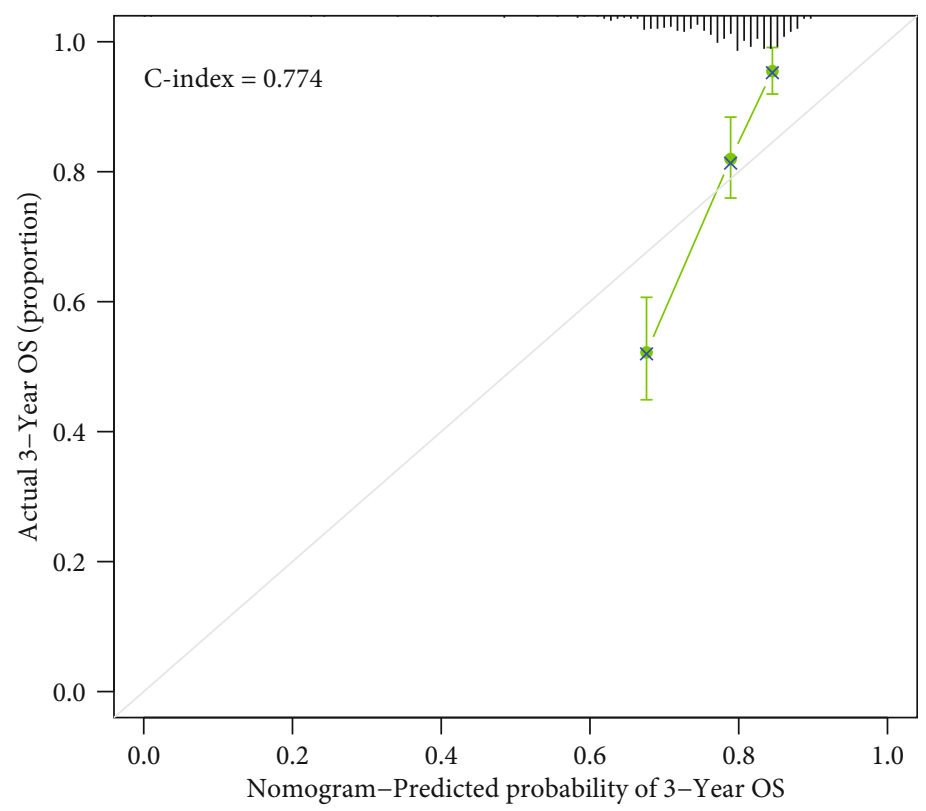

(i)

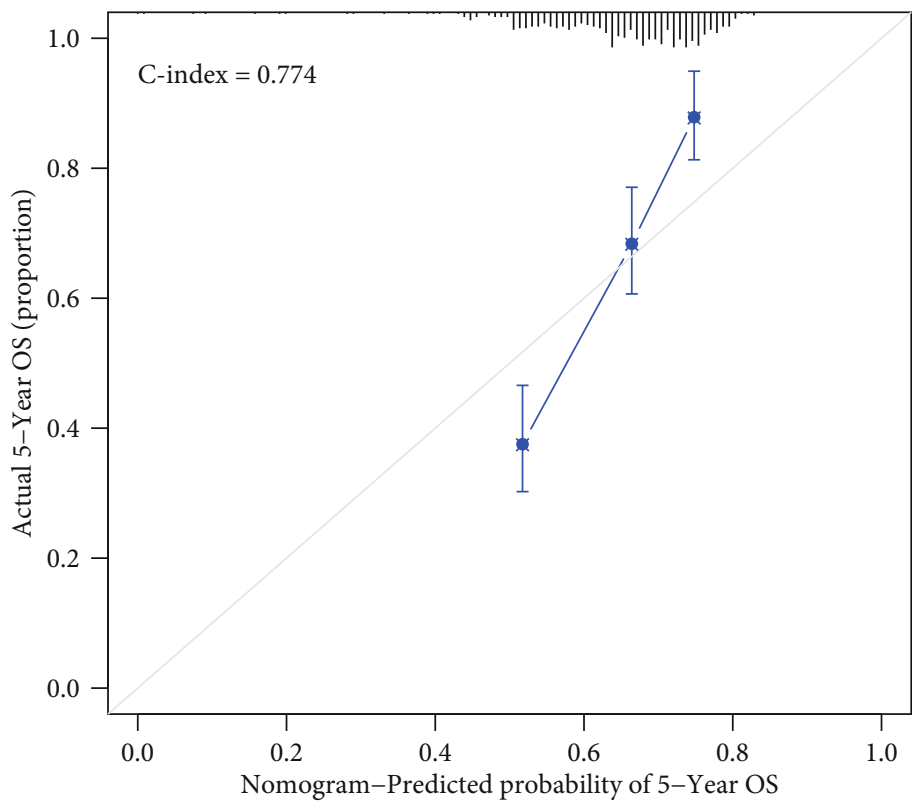

(j)

Figure 4: Nomogram and calibration curves. (a) Prognostic nomogram assembled from the training group to predict survival for ccRCC patients. (b, c, d) Calibration curves for the nomogram at 1-year, 3-year, and 5-year periods in the training group. (e, $f$, g) Calibration curves for the nomogram at 1-year, 3-year, and 5-year periods in the test group. (h, i, j) Calibration curves for the nomogram at 1-year, 3year, and 5-year periods in all samples.

\section{$-0.33 \cdot C O L C A 1-0.87 \cdot A C 027601.2-0.54 \cdot A C 004918.3-$ $0.11 \bullet S P I N T 1-A S 1$.}

3.4. Validation of the Survival-Predicting Signature. Having developed the nine redox-related lncRNA signature, all the samples both in the training group and test group acquired a risk score, and we set the medium value of the risk score in the training group as the cutoff to judge the risk level of patients as high risk or low risk (Figures 2(a), 2(c), 2(e)). Following this, survival analysis was performed to verify the survival-predicting availability of the signature (Figures 2(b), 2(d), 2(f)). Time-dependent ROC curve for 1 year, 3 years, and 5 years in the training group (Figures 3(a)$3(\mathrm{c})$ ), test group (Figures $3(\mathrm{~d})-3(\mathrm{f})$ ), and all samples (Figures 3(g)-3(i)) were drawn, and the AUC for the risk score in these three groups showed that risk score could act as an efficient prognostic factor even compared with other commonly used clinical prognostic factor. 


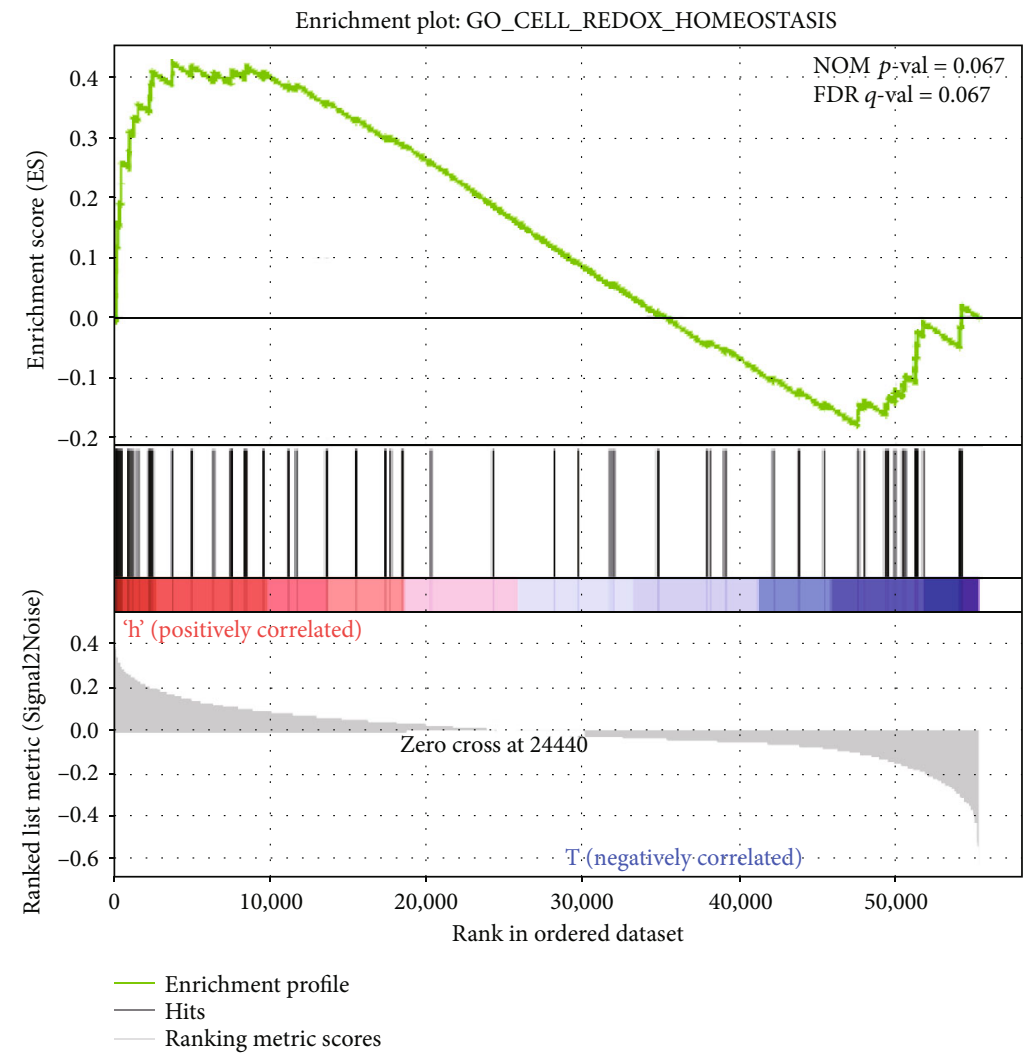

(a)

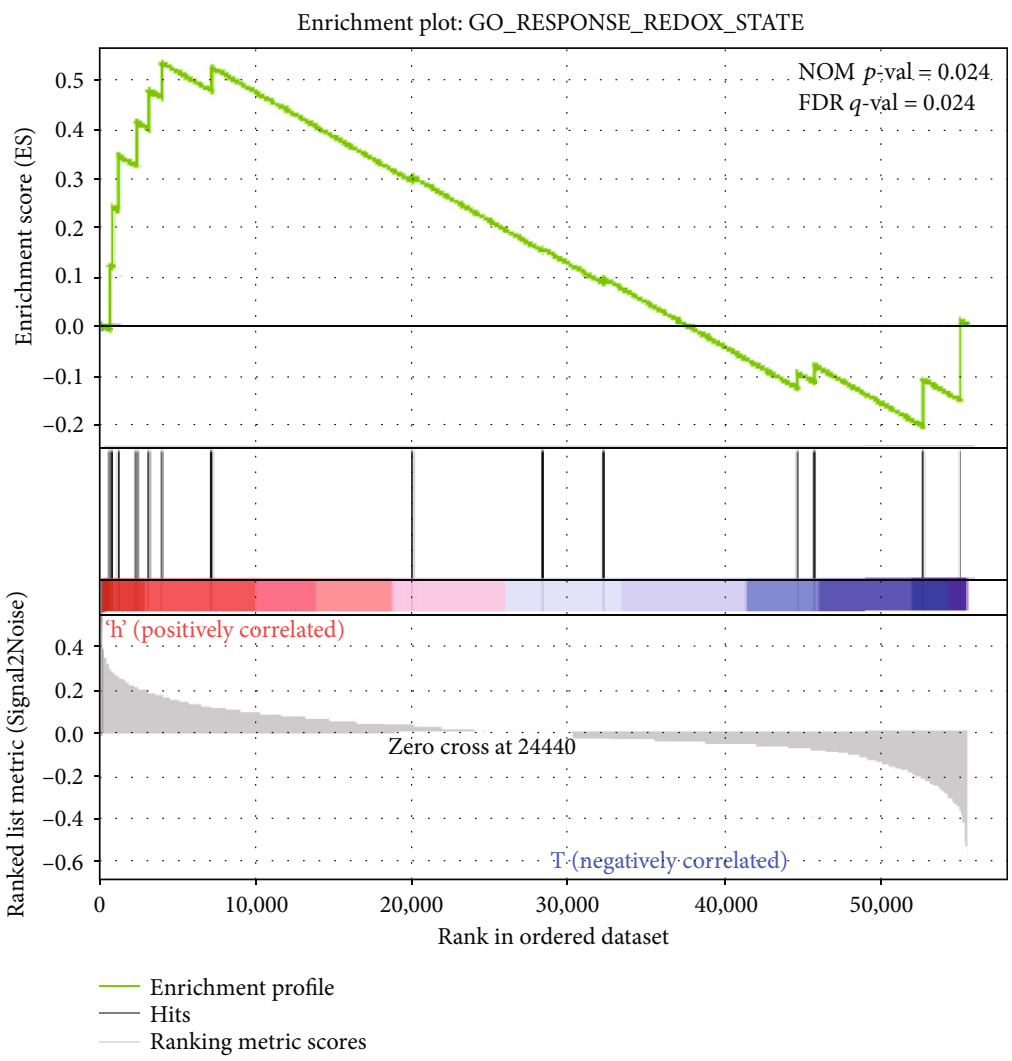

(b)

Figure 5: Continued. 


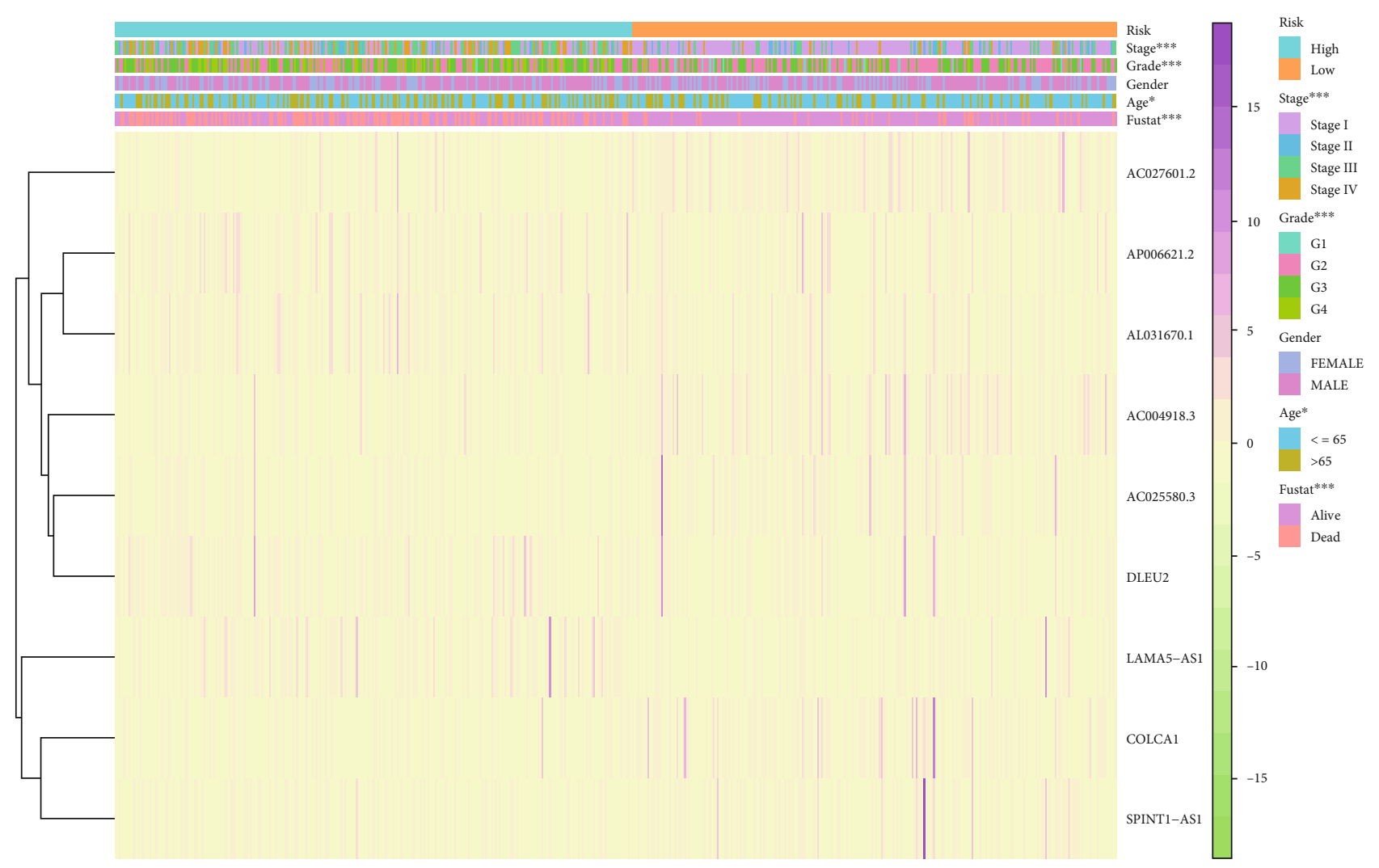

(c)

FIGURE 5: Gene set enrichment analysis (GSEA) of high-risk and low-risk ccRCC patients based on the redox-related lncRNA prognostic signature and expression heat map between high/low-risk patients. (a, b) The GSEA results show a significant enhancement of redoxrelated functions in the high-risk ccRCC patients. (c) Expression heat map for the nine redox-related lncRNAs in all samples.

\subsection{Construction and Validation of the Risk Score-Based} Nomogram. Having verified the efficacy of the signature, we would like to develop a more quantitative rather than qualitative approach for clinicians to predict the survival of the KIRC patients. Thus, we assembled a nomogram according to the risk score and clinicopathologic characteristics that contained age, gender, stage, and grade from the samples in the training group to predict the survival rate for 1 year, 3 years, and 5 years (Figure 4(a)). Also, the calibration curve for 1-year, 3-year, and 5-year survival rate in the training group (Figure 4(b)-4(d)), test group (Figure 4(e)-4(g)), and all KIRC samples (Figure $4(\mathrm{~h})-4(\mathrm{j})$ ) were plotted. Also, Cindex was calculated to assess the performance of the nomogram assembled according to the training group, and that was $0.782,0.766$, and 0.774 in the training group, test group, and all samples, which showed the perfect performance of the nomogram.

3.6. GSEA and Clinical Correlation. To explore the different redox-related functions in the high/low-risk group, we performed the enrichment analysis by using GSEA version 4.0.3 as shown in Figures 5(a) and 5(b). It showed that both two redox-related functions were enhanced in the high-risk group, of which the GO term response to the redox state was significantly enhanced in the high-risk group (NOM $p$ value $=0.024$, FDR $q$-value $=0.024)$, while the other
GO term cell redox homeostasis was not significant (NOM $p$ value $=0.065$, FDR $q$ value $=0.065$ ). In addition, the correlation between risk level and clinical characteristics and the differential expression of the nine redox-related lncRNAs in high/low risk were analyzed as shown Figure 5(c). It seemed age, grade, and stage were all significantly related to the risk level, which was also consistent with the outcome that high risk resulted in high mortality.

3.7. Coexpression Network, Correlation Plot, and Differential Expression Status. Finally, we focused on these nine redoxrelated lncRNAs about their coexpression and differential expression. The redox gene-redox-related lncRNA coexpression network was constructed (Figure 6(a)), and the Sankey plot (Figure $6(\mathrm{~b})$ ) showed that 4 of them were protective and the other 5 were risky. In addition, the correlation between the redox gene and redox-related lncRNA was plotted as the correlation network (Figure 7(a)) and correlation circle plot (Figure $7(\mathrm{~b})$ ). The differential expression status of the nine redox-related lncRNAs between normal/tumor tissue as in Figure 7 (c) showed that 7 of 9 redox-related lncRNAs were significantly high expression in the tumor tissue while the 2 left were significantly low expression in the tumor tissue. Besides, the correlation between the expression of these nine redox-related lncRNAs and the clinicopathological staging was explored and shown in Figure $7(\mathrm{~d})$. As 


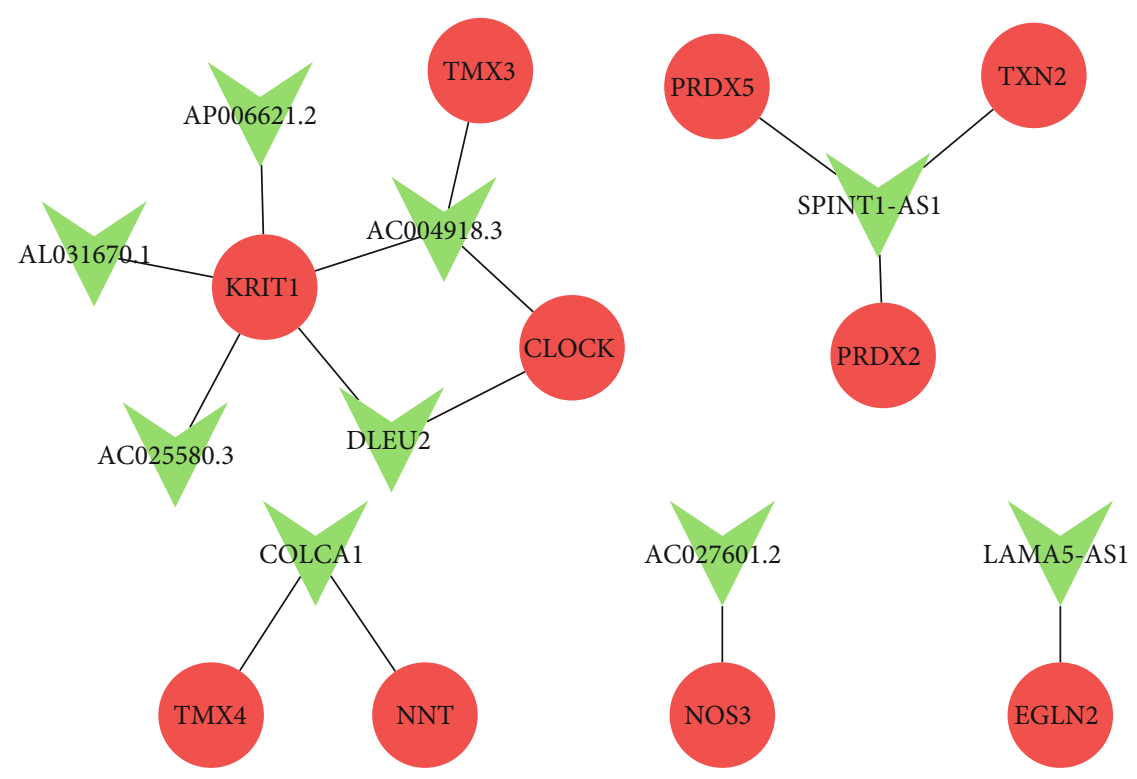

(a)
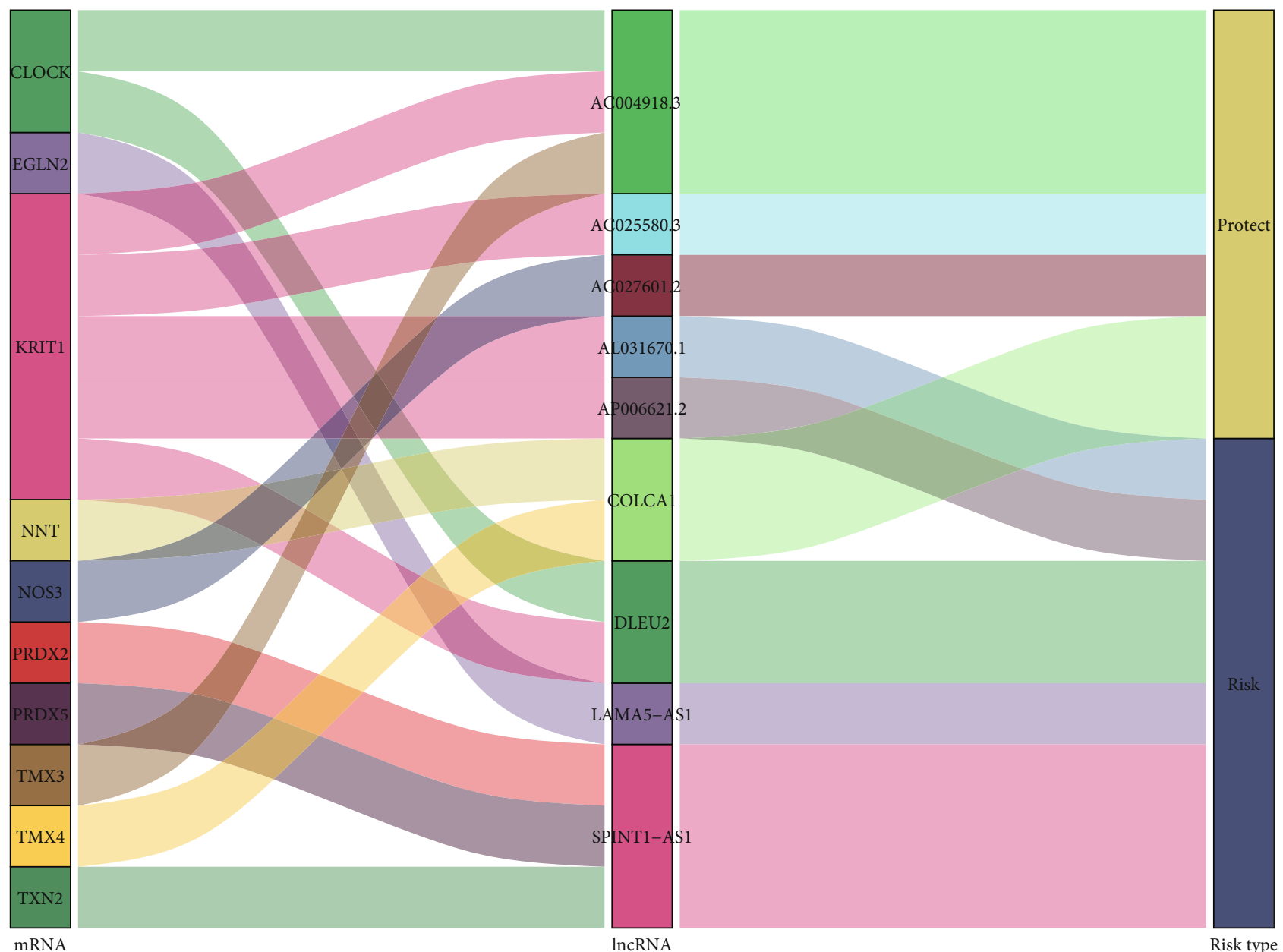

(b)

FIGURE 6: Redox gene-redox-related lncRNA interaction network and Sankey plot. (a) The interaction network between the redox-related lncRNA genes and redox-related protein-coding genes: red circle for redox-related mRNAs and green V-shape for redox-related lncRNAs. (b) In the Sankey plot for the interaction network, four of these redox-related lncRNAs were protective while the other five were indicative of risk. 


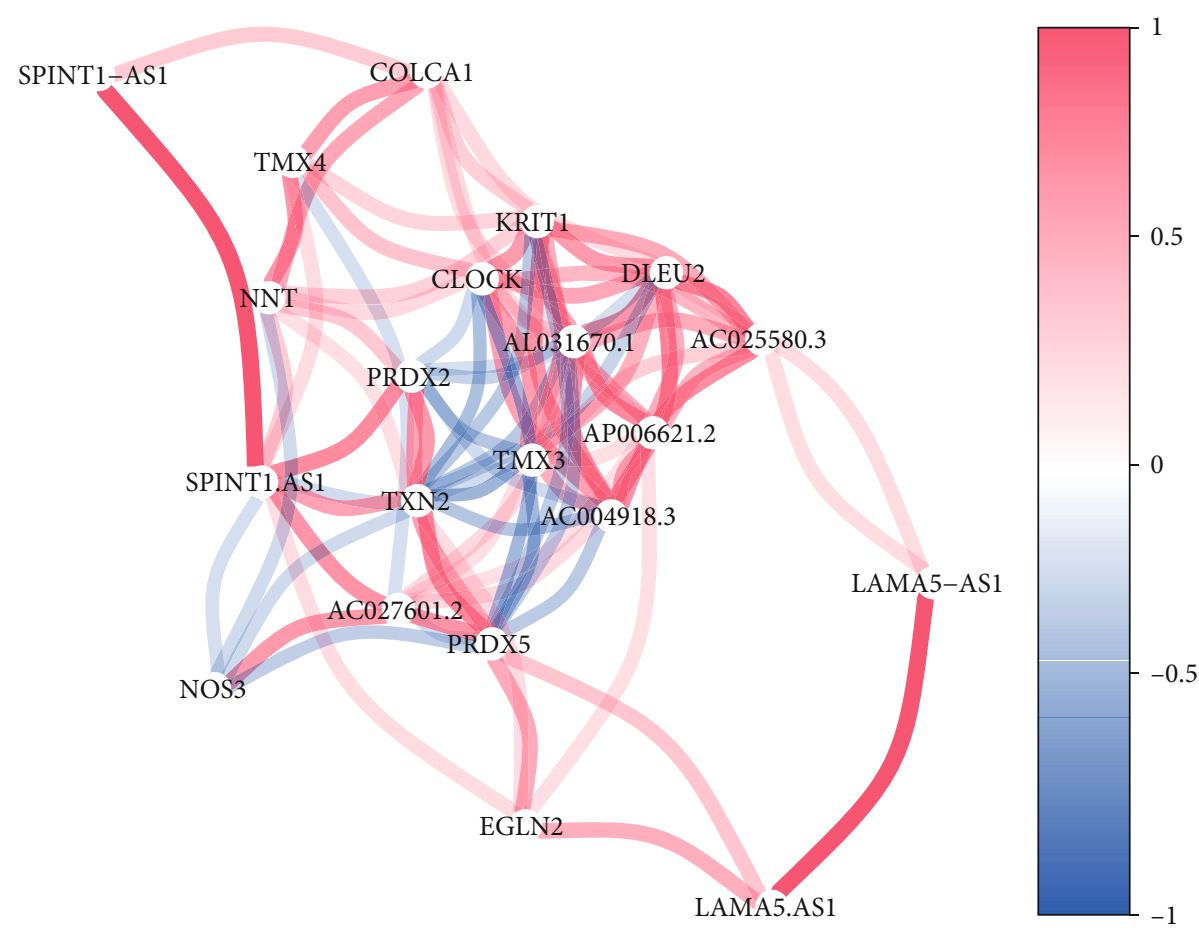

(a)

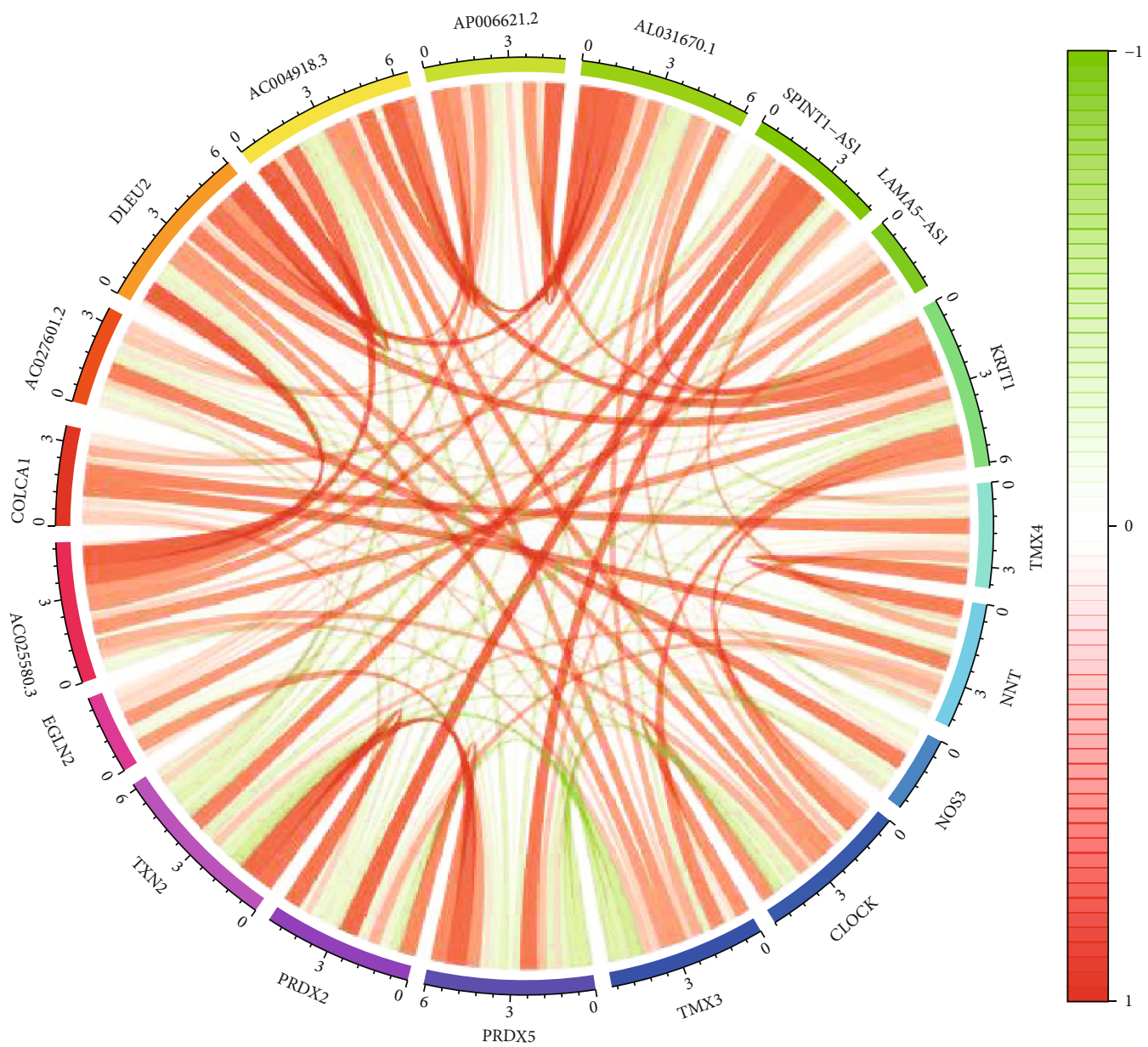

(b)

Figure 7: Continued. 


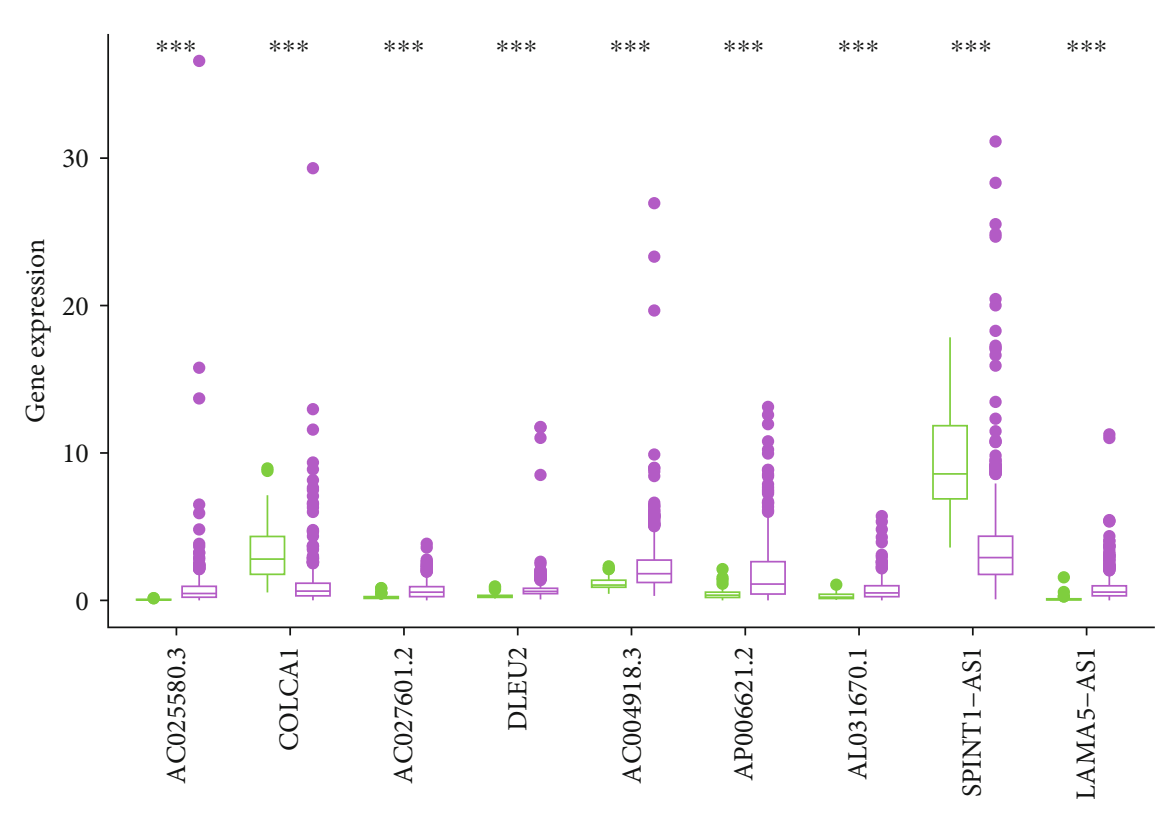

$$
\begin{aligned}
& \text { Type } \\
& \biguplus \text { Normal } \\
& \varsubsetneqq \text { Tumor }
\end{aligned}
$$

(c)

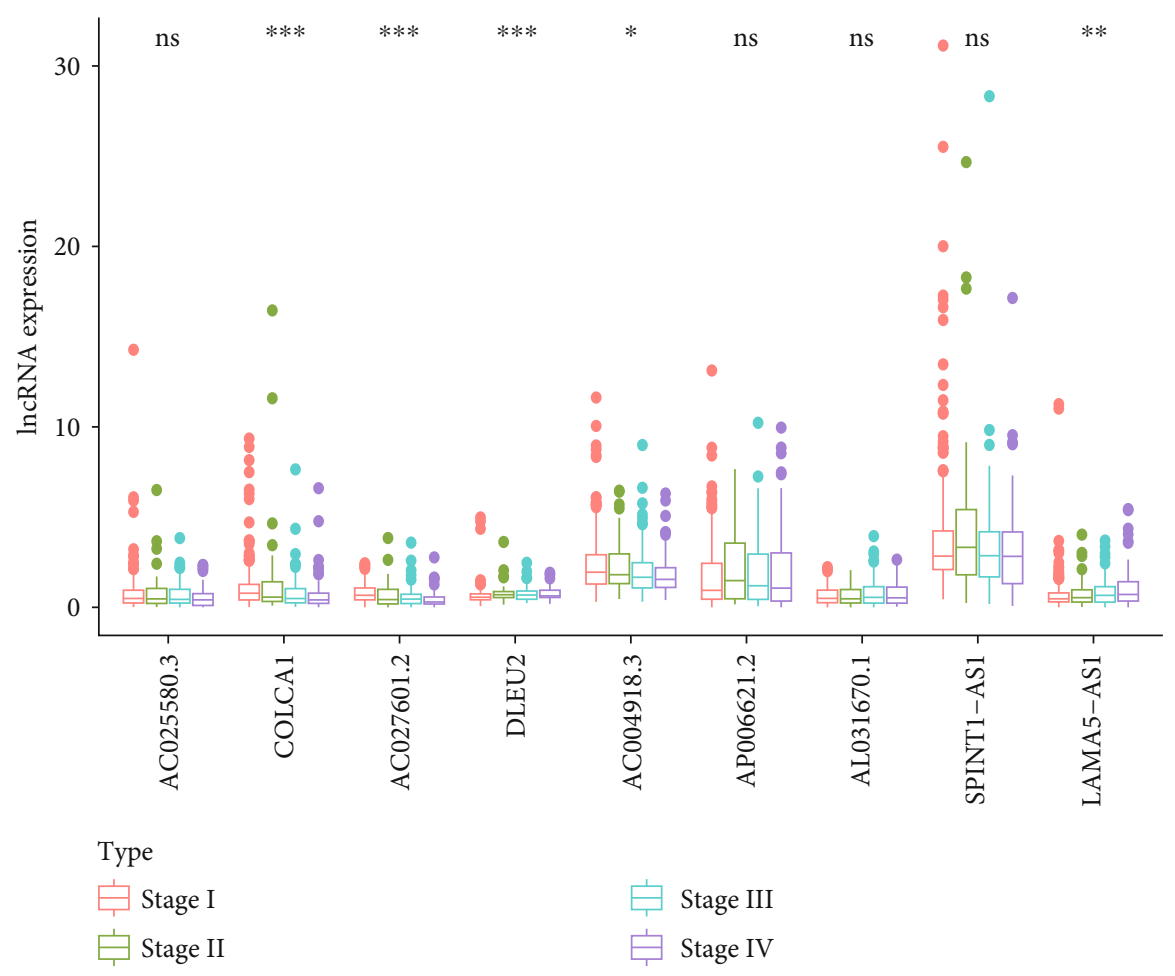

(d)

Figure 7: Correlation plot and the differential expression between normal/tumor tissue. (a) Correlation network for the redox gene and redox-related lncRNAs. (b) Circle plot for the correlation between redox gene and redox-related lncRNAs. (c) Differential expression for the nine redox-related lncRNAs between normal/tumor tissue. (d) Differential expression for the nine redox-related lncRNAs between stage I/II/III/IV patients. 


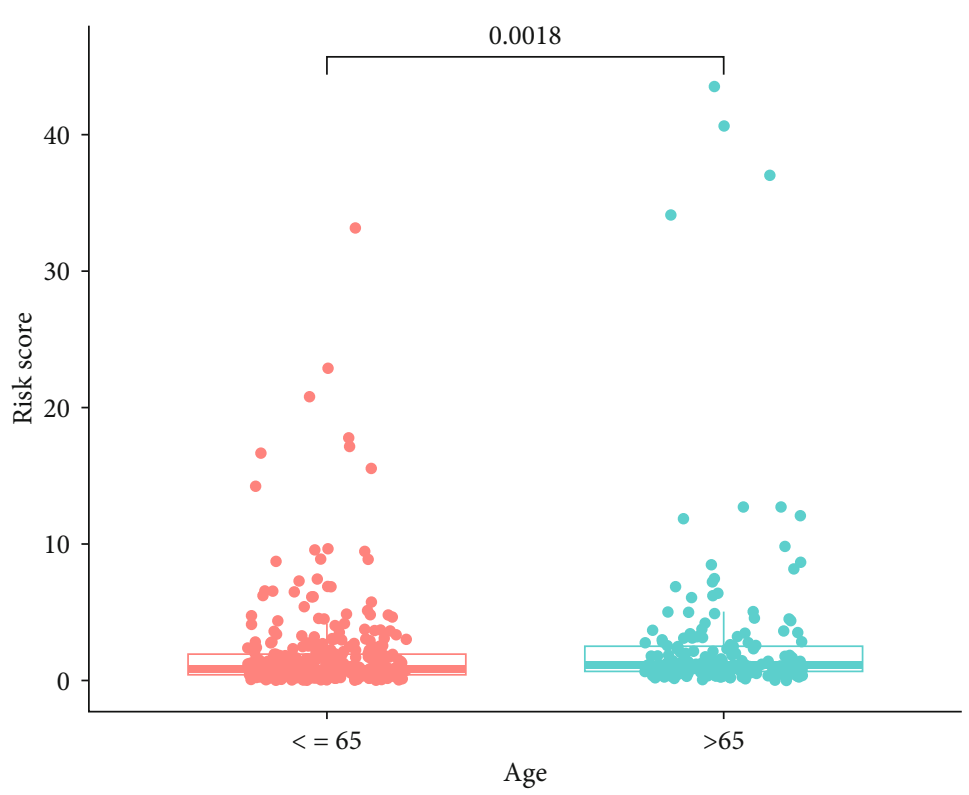

Age
$<>65$

(a)

Patients with age $<=65$

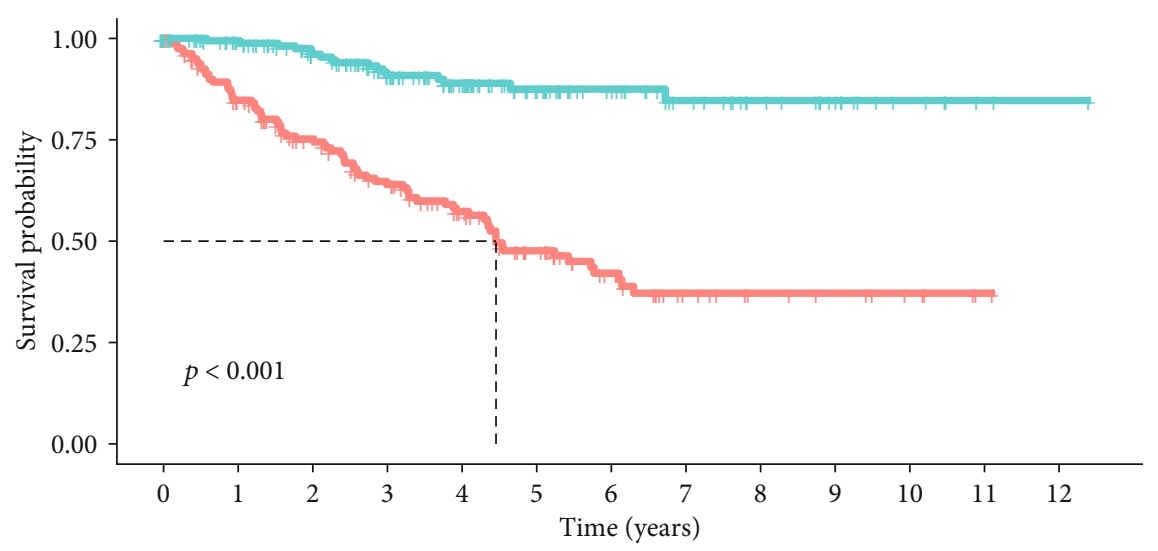

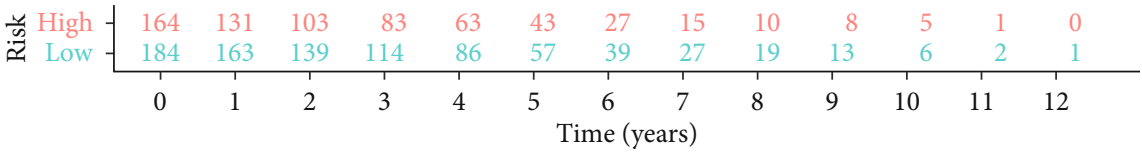

Risk

High

(b)

Figure 8: Continued. 
Patients with age $>65$
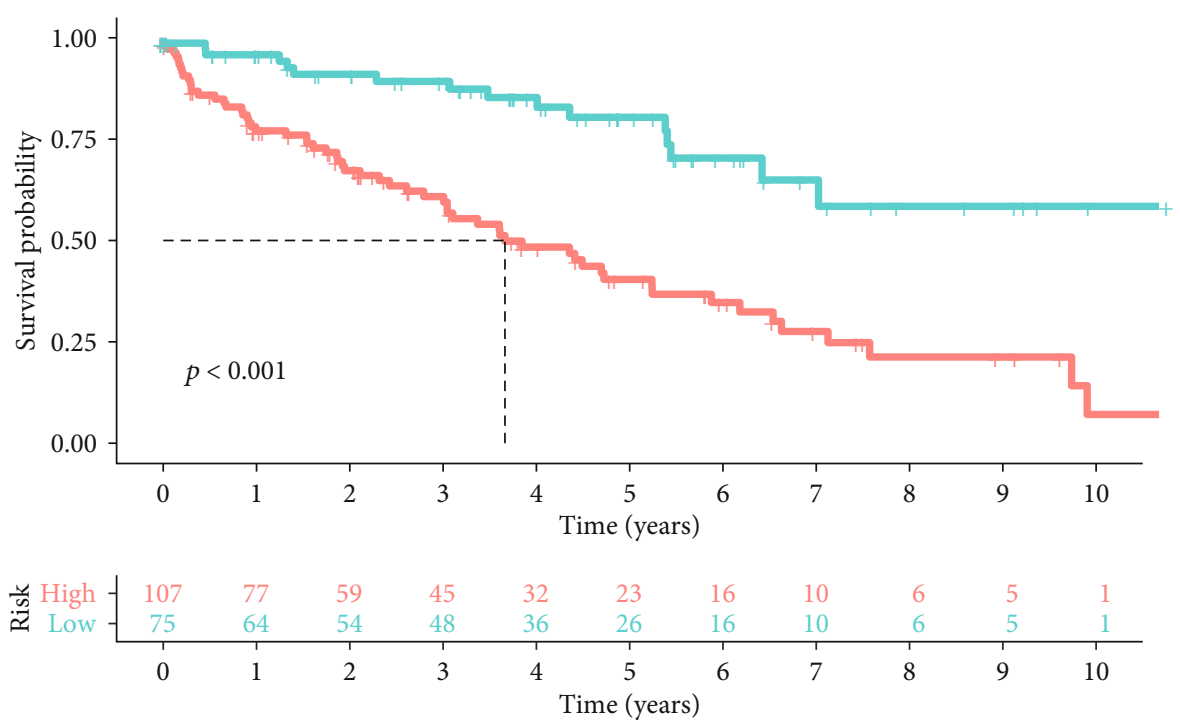

Risk

High

Low

(c)

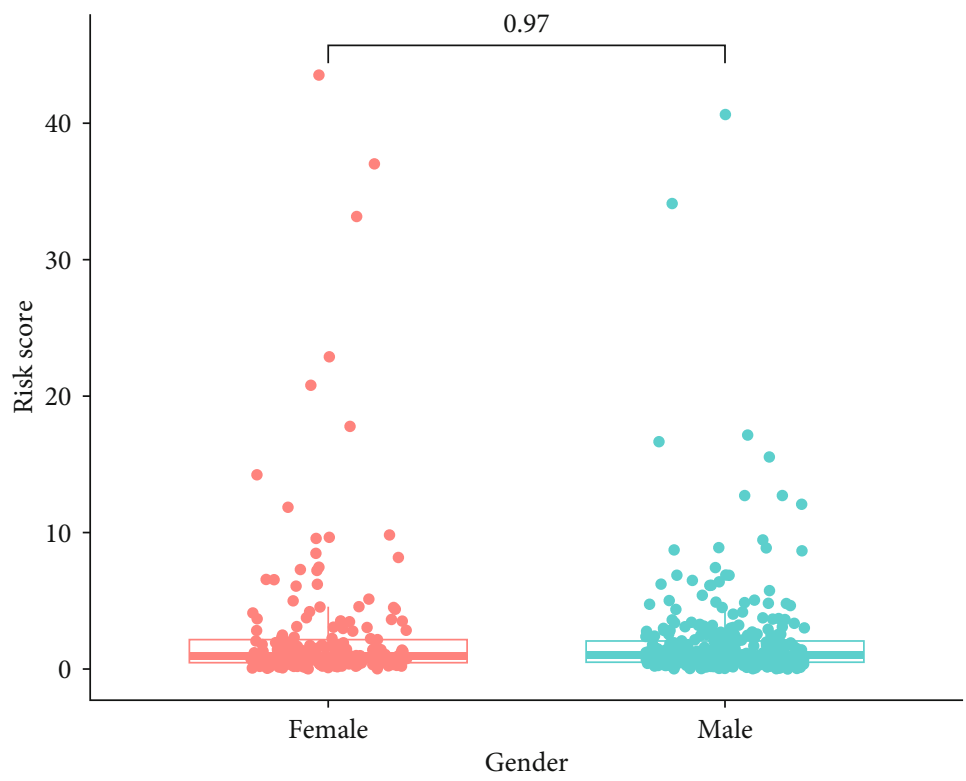

Gender

Female

Male

(d)

FIgure 8: Continued. 
Patients with FEMALE
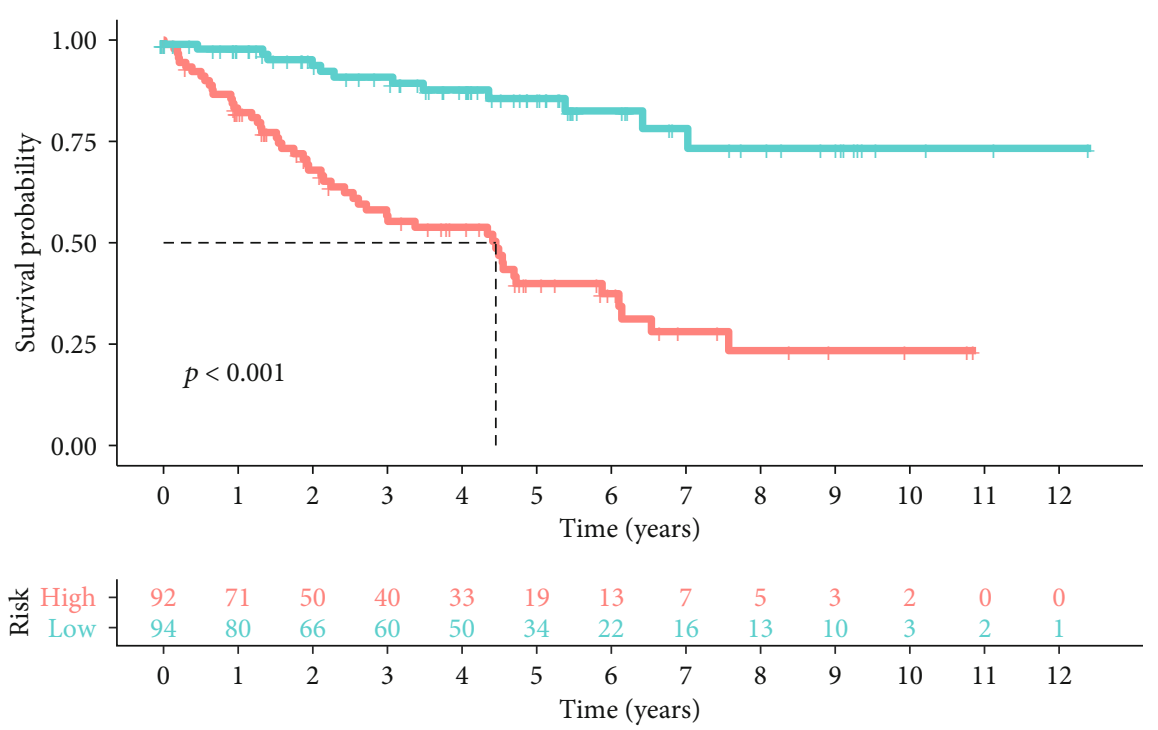

Risk

High

(e)

Patients with MALE

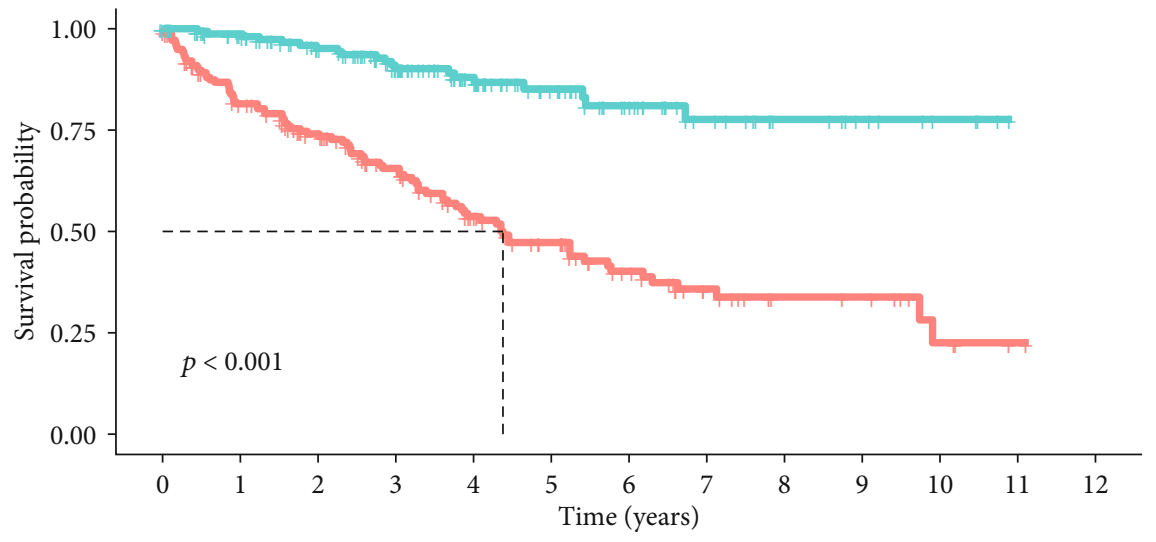

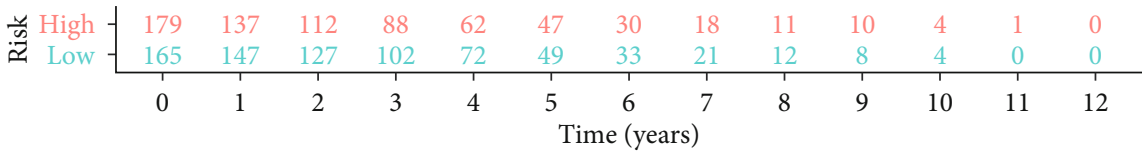

Risk

+ High

Low

(f)

Figure 8: Continued. 


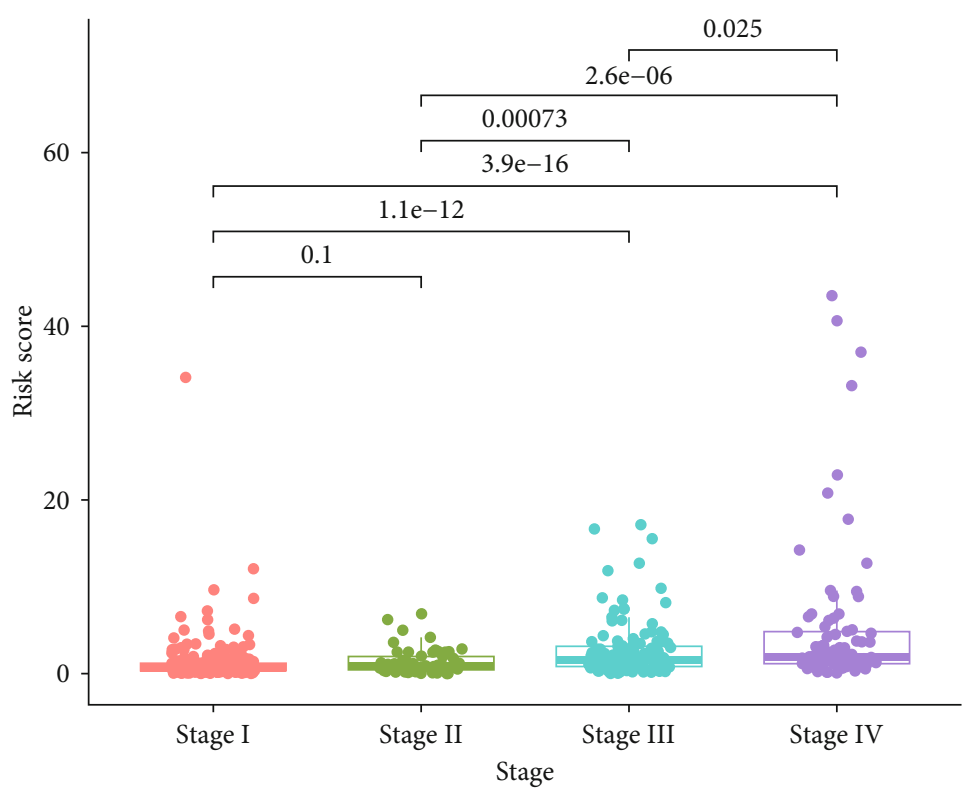

Stage

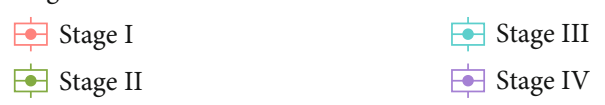

(g)

Patients with Stage I-II
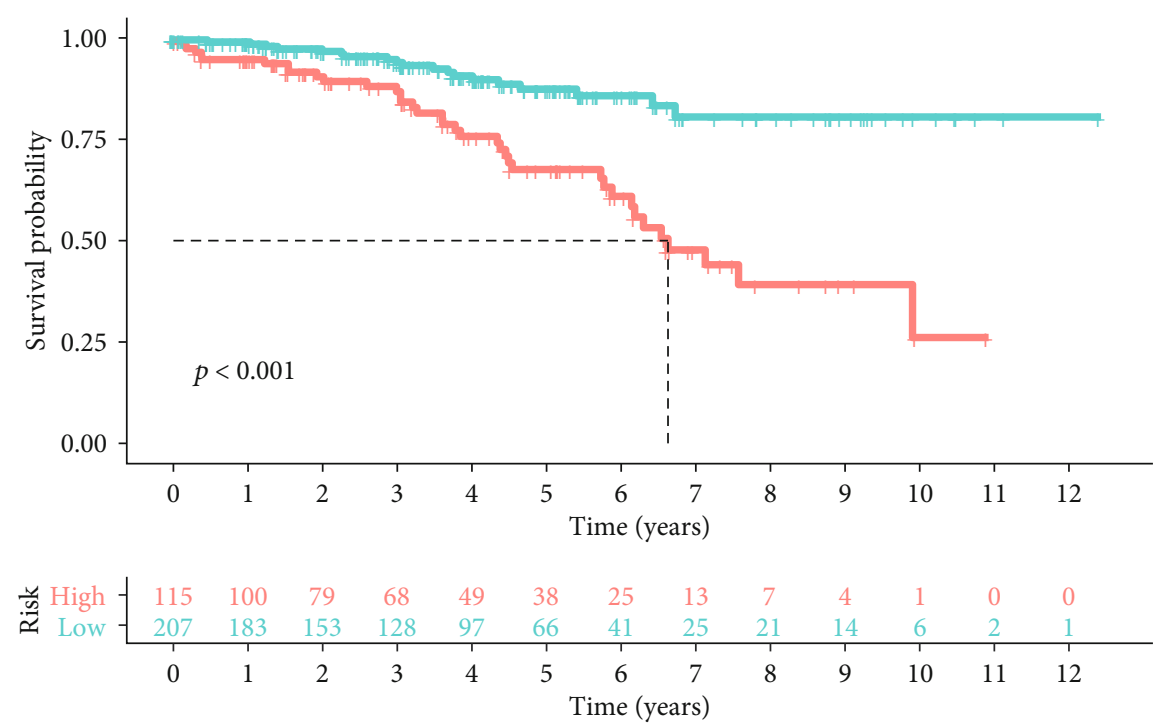

Risk

$\stackrel{1}{+}$ High

Low

(h)

Figure 8: Continued. 
Patients with Stage III-IV

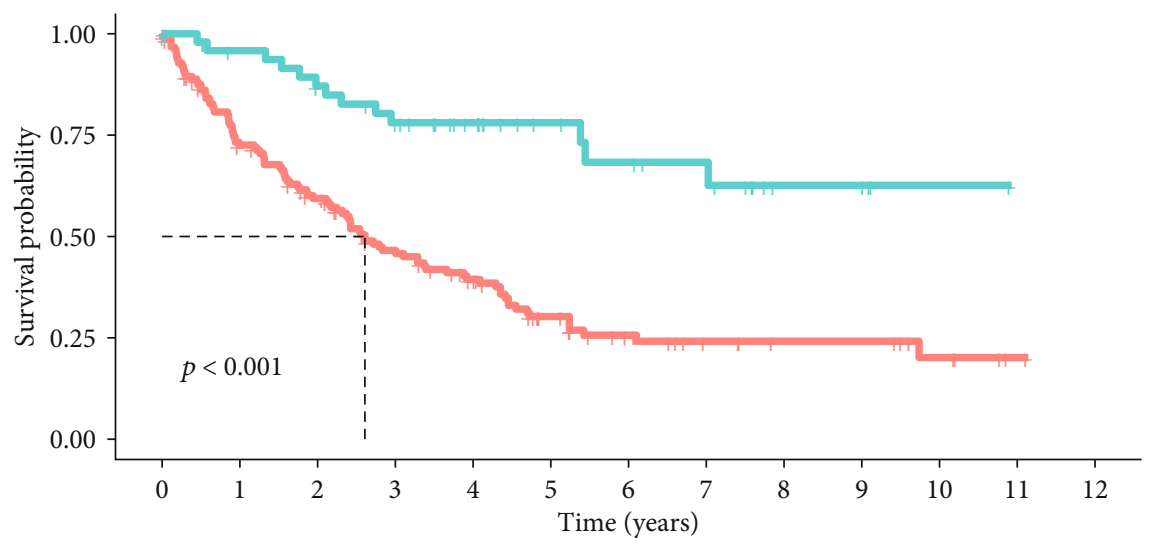

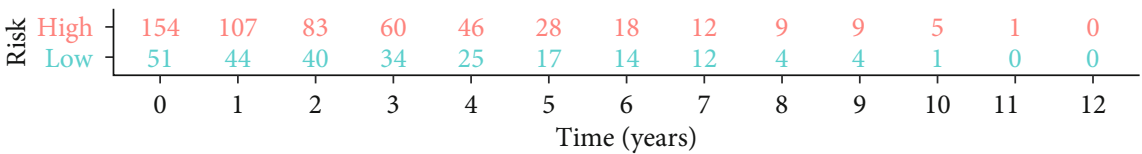

Risk

High

L Low

(i)

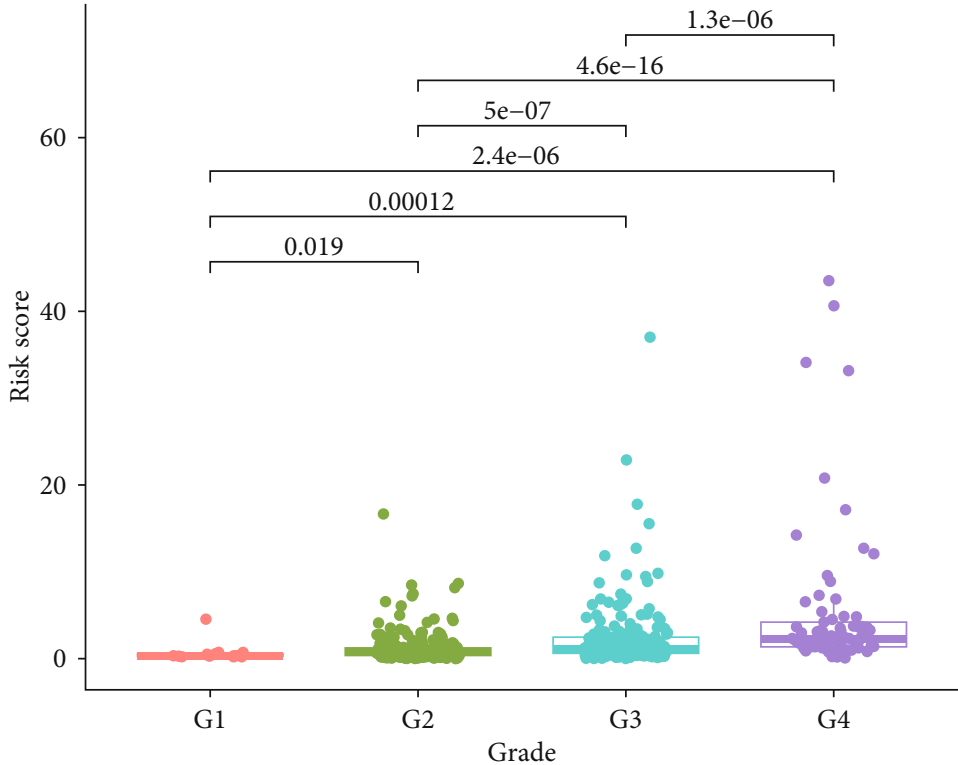

Grade

G1

G3

$-\mathrm{G} 2$

G4

(j)

Figure 8: Continued. 
Patients with G1-2

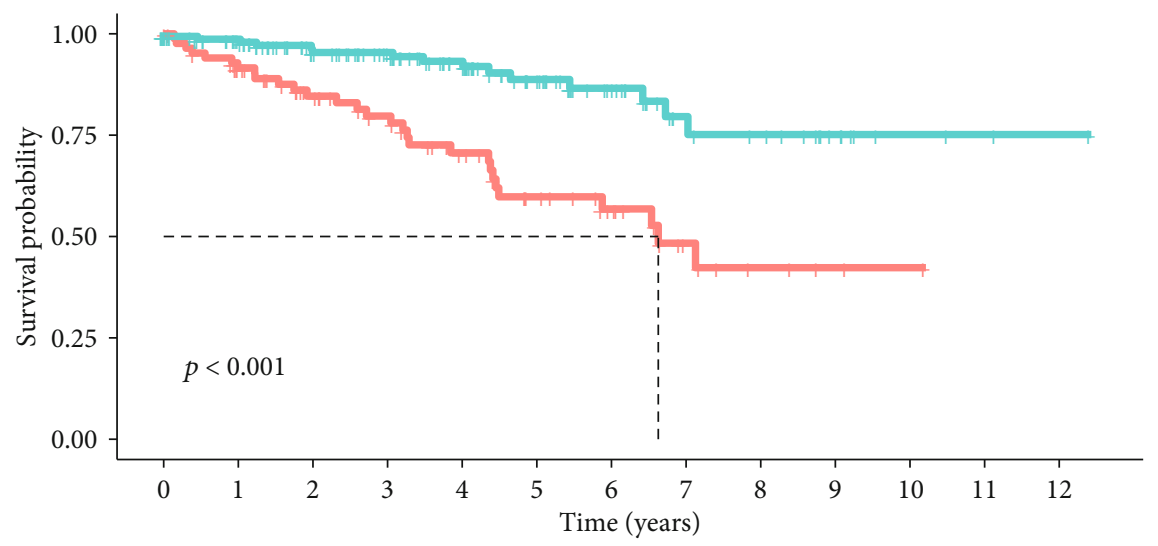

\begin{tabular}{|c|c|c|c|c|c|c|c|c|c|c|c|c|c|}
\hline $\begin{array}{l}\text { 뜨 High } \\
\text { क्ञ Low }\end{array}$ & $\begin{array}{c}86 \\
155 \\
\end{array}$ & $\begin{array}{r}73 \\
134 \\
\end{array}$ & $\begin{array}{c}56 \\
109 \\
\end{array}$ & $\begin{array}{l}47 \\
94 \\
\end{array}$ & $\begin{array}{l}35 \\
73 \\
\end{array}$ & $\begin{array}{l}24 \\
49\end{array}$ & $\begin{array}{l}17 \\
32 \\
\end{array}$ & $\begin{array}{c}8 \\
18 \\
\end{array}$ & $\begin{array}{r}4 \\
15 \\
\end{array}$ & $\begin{array}{l}2 \\
8 \\
\end{array}$ & $\begin{array}{l}1 \\
3 \\
\end{array}$ & $\begin{array}{l}0 \\
2 \\
\end{array}$ & $\begin{array}{l}0 \\
1 \\
\end{array}$ \\
\hline & 0 & 1 & 2 & 3 & 4 & 5 & 6 & 7 & 8 & 9 & 10 & 11 & 12 \\
\hline
\end{tabular}

Risk

+ High

1 Low

(k)

Patients with G3-4

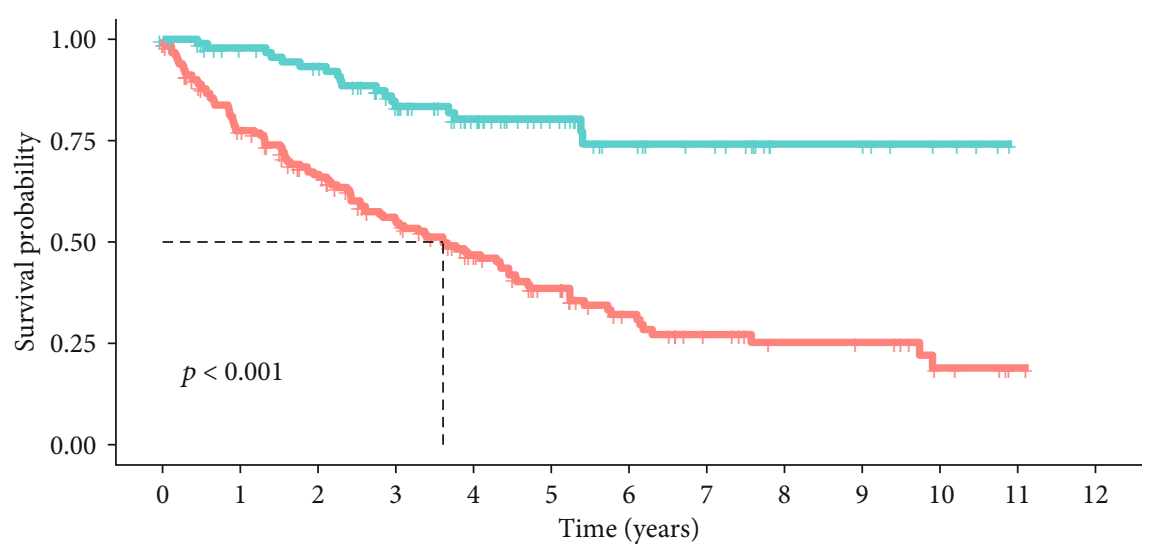

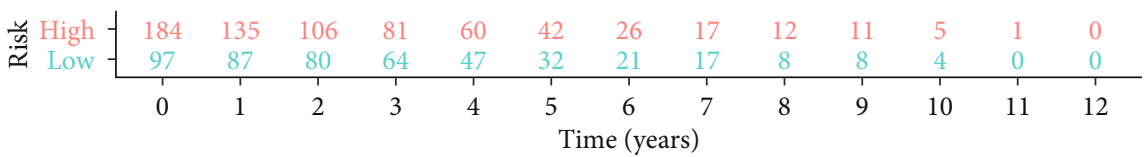

Risk

+ High

(1)

FIGURE 8: Difference in risk score between subgroups and further subgroup survival analysis. (a) Difference of risk score in patients with age $\leq 65$ or $>65$. (b) Risk score-based survival analysis in patients with age $\leq 65$. (c) Risk score-based survival analysis in patients with age $>$ 65. (d) Difference of risk score in female or male patients. (e) Risk score-based survival analysis in female patients. (f) Risk score-based survival analysis in male patients. (g) Difference of risk score in patients with different stage. (h) Risk score-based survival analysis in patients with stage I-II. (i) Risk score-based survival analysis in patients with stage III-IV. (j) Difference of risk score in patients with different grade. (k) Risk score-based survival analysis in patients with G1-2. (l) Risk score-based survival analysis in patients with G3-4.

for the differential expression status of the nine redox-related lncRNAs in different age, gender, and grade, the boxplot is shown in the supplementary file (available here).
3.8. Subgroup Analysis. In age subgroups, risk score in patients with age $>65$ was significantly higher than patients with age $\leq 65$ (Figure $8(\mathrm{a})$ ), and the prognostic signature 
was verified effective in both age $\leq 5$ (Figure $8(\mathrm{~b})$ ) and age $>65$ (Figure $8(\mathrm{c})$ ) subgroups. In the gender subgroup, there was no significant difference in the risk score between female and male patients (Figure $8(\mathrm{~d})$ ). And the prognostic signature was also effective in both female (Figure 8(e)) and male (Figure 8(f)) subgroups. As for the stage subgroups (Figure $8(\mathrm{~g})$ ), the risk score in stage III was significantly higher than that in stage II, and stage IV was significantly higher than stage III. However, stage II was higher than stage I with no significant difference. And the prognostic signature was effective in both the stage I-II (Figure 8(h)) and stage IIIIV (Figure 8(i)) subgroups. In the grade subgroups, the risk score was significantly increased between G1 and G2 and G3 and G4 (Figure 8(j)), and the prognostic signature was still effective in both the G1-2 (Figure 8(k)) and G3-4 (Figure 8(1)) subgroups.

\section{Discussion}

ccRCC is the most common type of RCC in humans. With the development of clinical management of ccRCC, several prognostic factors, such as tumor grade and stage, tumor size, and tumor number, are well characterized. However, ccRCC has complex genetic and molecular alterations [24], which could affect the biological processes, and some of the biological processes are closely associated with the prognosis of ccRCC patients, such as autophagy [25], ferroptosis [15], and redox [26]. Most of these commonly used prognostic factors do not consider either genetic and molecular alterations or dysregulated biological processes, and it made these commonly used prognostic factors not perfect for accurate prognostic prediction of ccRCC patients [27]. As an emerging genetic and molecular biomarker, lncRNA is a new class of noncoding RNA molecules that regulate cancer cell growth, progression, and survival [28]. Therefore, it is necessary to establish a lncRNA signature to predict the prognosis of ccRCC patients.

In this study, we focused on the redox process and constructed a nine redox-related lncRNA prognostic signature $($ Risk score $=1.23 \bullet D L E U 2+0.21 \bullet A P 006621.2+0.89 \bullet A$ $L 031670.1+0.26 \bullet L A M A 5-A S 1-0.56 \bullet A C 025580.3-0.33 \bullet$ COLCA1 - 0.87•AC027601.2 - 0.54•AC004918.3 - 0.11•SPI $N T 1-A S 1$.) in the training group by the LASSO regression and COX regression, which considered both molecular alteration and dysregulated biological process. Meanwhile, $\chi^{2}$-test or Fisher's exact test found the nine redox-related lncRNA signature was significantly related to tumor grade, stage, patients' age, and survival status of ccRCC patients. In addition, risk analysis, survival analysis, and 1-year, 3-year, 5 -year multivariate ROC in both the training group and test group well verified the efficacy of the survival-predicting signature. Then, a concise nomogram consisted of the nine redox-related lncRNA signature, age, gender, grade, and stage was developed from the data in the training group for prognostic prediction of ccRCC patients; both internal cross-validation and external set validation showed great effectiveness, and the calibration curve showed great convergency to the standard curve. Further subgroup analysis verified the effectiveness of our prognostic signature and indicated the universality of this prognostic signature. Finally, having verified the effectiveness of our nine-redox-related IncRNA signature, we focused on the interaction between the redox genes and these nine redox-related lncRNAs, constructed a redox gene-lncRNA interaction network, and performed a GSEA analysis to explore the differences in redox functions between high/low risk. Interestingly, we found both GO CELL REDOX HOMEOSTASIS and GO RESPONSE TO REDOX STATE were enhanced in the high-risk group, which was consistent with the previous study that high redox level in cancer could influence the survival of tumor patients by initiating/stimulating tumorigenesis and supporting transformation/proliferation of cancer cells or causing cell death [29].

In this signature which consisted of nine prognostic lncRNAs related to redox genes, COLCA1 has been reported and identified as a key lncRNA in colorectal cancer [30-32], and DLEU2 has been reported related to the development of multiple cancers [33-36]. Chen et al. reported that lncRNA DLEU2 could regulate $m i R-30 a-5 p$ and related to the aggressiveness of ccRCC [37]. SPINT1-AS1 has been reported as a prognostic factor in esophageal squamous cell carcinoma and colorectal cancer [38, 39]. Xiang et al. also reported SPINT1-AS1 as a crucial factor for pan-cancer cell sensitivity to lapatinib [40]. LAMA5-AS1 has been reported as a significant factor in the pathogenesis of multiple myeloma [41]. As for the other 5 lncRNAs, there were few reports about them.

Redox plays an essential role in the pathogeneses and progression of tumors. Regulation of reactive oxygen species (ROS) production is crucial in highly proliferative cancer cells, owing to the presence of oncogenic mutations that promote aberrant metabolism and gene expression [42]. Cancer cells can produce ROS, which diffuses into the tumor microenvironment, then initiates stromal oxidative stress and autophagy, and leads to angiogenesis. Sosa et al. [43] revealed that cancer cells develop resistance to ROS by inducing a new redox balance, which further results in cellular adaptation and proliferation under increased oxidative pressure. Indeed, the imbalance of the redox homeostasis system is closely related to the RCC occurrence and progression. In RCC patients, cytosolic antioxidant enzyme activities are shown to be decreased [44]. In recent years, redox balance has been reported regulated by long noncoding RNAs [45]; more and more researchers developed to identify the significant lncRNA-redox regulation network. Chen et al. reported that lncRNA GAS5 regulated the redox balance and dysregulates the cell cycle and apoptosis in malignant melanoma cells [46]. He et al. reported lncRNA MACC1-AS1 promoted stemness and chemoresistance through fatty acid oxidation in gastric cancer [47]. Here, our works contributed to further the comprehension of these nine redox-related lncRNAs and their interaction with redox balance, which might provide potential targets for the treatment in the future.

However, our study still has some limitations. First, the training group and test group are both obtained from TCGA, and it would be better if there is an independent cohort as an external validation set. In addition, we did not define the mechanisms behind the lncRNA-based signature's mediation of redox in the initiation and progression of ccRCC. Despite these limitations, this is the first redox-related lncRNA-based 
survival-predicting signature, and our nomogram provides a quantitative approach for clinicians to predict survival, which can easily separate patients with poor prognosis from all the ccRCC patients by performing PCR. Then, clinicians can perform more individualized treatment regimens for patients with different prognosis, which will contribute to individual treatment and save more public health resources. Meanwhile, this nomogram consisted of objective indicators, which can reduce the interobservers' differences and more accurately predict survival.

\section{Conclusions}

In summary, we successfully developed and verified a nineredox-related lncRNA signature that could predict the overall survival of ccRCC patients. The prognostic signature proved superior compared to the other common prognostic factors. We further assembled a nomogram connecting this signature with clinicopathologic characteristics for 1-, 3-, and 5-year OS, which can provide clinicians with a quantitative rather than qualitative approach in predicting ccRCC survival. This will help clinicians make treatment decisions more easily and accurately in the future. It is, however, necessary to carry out a large-scale, multicenter prospective research to confirm our results.

\section{Data Availability}

The source data of this study were derived from the public repositories, as indicated in the section of "Materials and Methods" of the manuscript. And all data that support the findings of this study are available from the corresponding author upon reasonable request.

\section{Conflicts of Interest}

The authors declare that they have no conflicts of interest.

\section{Authors' Contributions}

XQD and XY contributed to the design, analysis, and interpretation of data; drafting of the manuscript; and critical revision of the manuscript. XQD and LJL contributed to the statistical analysis. XQD, XY, LJL, LCQ, SJX, LC, and WSG contributed to the methodology. LCQ, SJX, LC, and WSG contributed to the project administration. XQD and $\mathrm{XY}$ wrote the manuscript (original draft). XQD, LJL, LCQ, SJX, LC, and WSG wrote the manuscript (review and editing). Qi-Dong, Xia; Yang, Xun; Cong Li; and Shao-Gang Wang contributed equally to this work.

\section{Acknowledgments}

We thank all the R programming package developers.

\section{Supplementary Materials}

Supplementary file. Differential expression of the nine redoxrelated lncRNAs in different groups. S1. Differential expression between age $\leq 65$ and age $>65$. S2. Differential expression between male and female. S3. Differential expression between different pathological grade. (Supplementary Materials)

\section{References}

[1] H. Moch, A. L. Cubilla, P. A. Humphrey, V. E. Reuter, and T. M. Ulbright, "The 2016 WHO classification of tumours of the urinary system and male genital organs-part a: renal, penile, and testicular tumours," European Urology, vol. 70, no. 1, pp. 93-105, 2016.

[2] R. L. Siegel, K. D. Miller, and A. Jemal, "Cancer statistics, 2020," CA: a Cancer Journal for Clinicians, vol. 70, no. 1, pp. 7-30, 2020

[3] U. Capitanio and F. Montorsi, "Renal Cancer," The Lancet, vol. 387, no. 10021, pp. 894-906, 2016.

[4] E. Jonasch, J. Gao, and W. K. Rathmell, "Renal cell carcinoma," BMJ, vol. 349, 2014.

[5] M. B. Atkins and N. M. Tannir, "Current and emerging therapies for first-line treatment of metastatic clear cell renal cell carcinoma," Cancer Treatment Reviews, vol. 70, pp. 127-137, 2018.

[6] T. K. Choueiri, B. Escudier, T. Powles et al., "Cabozantinib versus everolimus in advanced renal cell carcinoma (METEOR): final results from a randomised, open-label, phase 3 trial," The Lancet Oncology, vol. 17, no. 7, pp. 917-927, 2016.

[7] R. J. Motzer, B. Escudier, D. F. McDermott et al., "Nivolumab versus everolimus in advanced renal-cell carcinoma," The New England Journal of Medicine, vol. 373, no. 19, pp. 1803-1813, 2015.

[8] R. J. Motzer, T. Powles, M. B. Atkins et al., "IMmotion151: a randomized phase III study of atezolizumab plus bevacizumab vs sunitinib in untreated metastatic renal cell carcinoma," in Abstract (588) presented at the annual genitourinary cancers symposium of the American Society of Clinical Oncology, San Francisco, CA, February 2018.

[9] V. Ficarra, A. Galfano, M. Mancini, G. Martignoni, and W. Artibani, "TNM staging system for renal-cell carcinoma: current status and future perspectives," The Lancet Oncology, vol. 8, no. 6, pp. 554-558, 2007.

[10] S. You, Y. Zhang, J. Xu et al., "The role of BRG1 in antioxidant and redox signaling," Oxidative Medicine and Cellular Longevity, vol. 2020, 12 pages, 2020.

[11] K. M. Holmström and T. Finkel, "Cellular mechanisms and physiological consequences of redox-dependent signalling," Nature Reviews Molecular Cell Biology, vol. 15, no. 6, pp. 411-421, 2014.

[12] S. Reuter, S. C. Gupta, M. M. Chaturvedi, and B. B. Aggarwal, "Oxidative stress, inflammation, and cancer: how are they linked?," Free Radical Biology and Medicine, vol. 49, no. 11, pp. 1603-1616, 2010.

[13] D. Zhou, L. Shao, and D. R. Spitz, "Reactive oxygen species in normal and tumor stem cells," in Advances in Cancer Research, pp. 1-67, Academic Press Inc., 2014.

[14] J. G. Costa, N. Saraiva, I. Batinic-Haberle, M. Castro, N. G. Oliveira, and A. S. Fernandes, "The SOD mimic MnTnHex-2pyp5+ reduces the viability and migration of 786-O human renal cancer cells," Antioxidants, vol. 8, no. 10, p. 490, 2019.

[15] H. Miess, B. Dankworth, A. M. Gouw et al., "The glutathione redox system is essential to prevent ferroptosis caused by impaired lipid metabolism in clear cell renal cell carcinoma," Oncogene, vol. 37, no. 40, pp. 5435-5450, 2018. 
[16] Y. Xiao, T. Xiao, W. Ou et al., "LncRNA SNHG16 as a potential biomarker and therapeutic target in human cancers," Biomarker Research, vol. 8, no. 1, 2020.

[17] M. Guttman and J. L. Rinn, "Modular regulatory principles of large non-coding RNAs," Nature, vol. 482, no. 7385, pp. 339346, 2012.

[18] F. Kopp and J. T. Mendell, "Functional classification and experimental dissection of long noncoding RNAs," Cell, vol. 172, no. 3, pp. 393-407, 2018.

[19] J. J. Quinn and H. Y. Chang, "Unique features of long noncoding RNA biogenesis and function," Nature Reviews Genetics, vol. 17, no. 1, pp. 47-62, 2016.

[20] W. Jiang, Q. Guo, C. Wang, and Y. Zhu, "A nomogram based on 9-lncRNAs signature for improving prognostic prediction of clear cell renal cell carcinoma," Cancer Cell International, vol. 19, no. 1, 2019.

[21] Y. J. Su, J. Yu, Y. Q. Huang, and J. Yang, "Circulating long noncoding RNA as a potential target for prostate cancer," International Journal of Molecular Sciences, vol. 16, no. 12, pp. 13322-13338, 2015.

[22] L. Nandagopal and G. Sonpavde, "Circulating biomarkers in bladder cancer," Bladder Cancer, vol. 2, no. 4, pp. 369-379, 2016.

[23] X. Mao, X. Qin, L. Li et al., “A 15-long non-coding RNA signature to improve prognosis prediction of cervical squamous cell carcinoma," Gynecologic Oncology, vol. 149, no. 1, pp. 181$187,2018$.

[24] The Cancer Genome Atlas Research Network, "Comprehensive molecular characterization of clear cell renal cell carcinoma," Nature, vol. 499, no. 7456, pp. 43-49, 2013.

[25] T. M. Jones, J. S. Carew, and S. T. Nawrocki, "Therapeutic targeting of autophagy for renal cell carcinoma therapy," Cancers (Basel), vol. 12, no. 5, article 1185, 2020.

[26] T. Radic, V. Coric, Z. Bukumiric et al., "GSTO1 * CC genotype (Rs4925) predicts shorter survival in clear cell renal cell carcinoma male patients," Cancers (Basel), vol. 11, no. 12, article 2038, 2019.

[27] H. Moch, W. Artibani, B. Delahunt et al., "Reassessing the current UICC/AJCC TNM staging for renal cell carcinoma," European Urology, vol. 56, no. 4, pp. 636-643, 2009.

[28] E. S. Martens-Uzunova, R. Böttcher, C. M. Croce, G. Jenster, T. Visakorpi, and G. A. Calin, "Long noncoding RNA in prostate, bladder, and kidney cancer," European Urology, vol. 65, no. 6, pp. 1140-1151, 2014.

[29] J. D. Hayes, A. T. Dinkova-Kostova, and K. D. Tew, "Oxidative stress in cancer," Cancer Cell, vol. 38, no. 2, pp. 167-197, 2020.

[30] N. Song, K. Kim, A. Shin et al., "Colorectal cancer susceptibility loci and influence on survival," Genes, Chromosomes and Cancer, vol. 57, no. 12, pp. 630-637, 2018.

[31] V. D. Peltekova, M. Lemire, A. M. Qazi et al., "Identification of genes expressed by immune cells of the colon that are regulated by colorectal cancer-associated variants," International Journal of Cancer, vol. 134, no. 10, pp. 2330-2341, 2014.

[32] A. Closa, D. Cordero, R. Sanz-Pamplona et al., "Identification of candidate susceptibility genes for colorectal cancer through eQTL analysis," Carcinogenesis, vol. 35, no. 9, pp. 2039-2046, 2014.

[33] V. K. Srinivasan, S. Naseem, N. Varma, D. P. Lad, and P. Malhotra, "Genomic alterations in chronic lymphocytic leukemia and their correlation with clinico-hematological param- eters and disease progression," Blood Research, vol. 55, no. 3, pp. 131-138, 2020.

[34] W. Wu, Y. Zhao, E. Gao et al., "LncRNA DLEU2 accelerates the tumorigenesis and invasion of non-small cell lung cancer by sponging miR-30a-5p," Journal of Cellular and Molecular Medicine, vol. 24, no. 1, pp. 441-450, 2020.

[35] X. Li, F. Xu, Q. Meng et al., "Long noncoding RNA DLEU2 predicts a poor prognosis and enhances malignant properties in laryngeal squamous cell carcinoma through the miR-30c5p/PIK3CD/Akt axis," Cell Death \& Disease, vol. 11, no. 6, p. 472, 2020.

[36] T. Lu, R. Wang, H. Cai, and Y. Cui, "Long non-coding RNA DLEU2 promotes the progression of esophageal cancer through miR-30e-5p/E2F7 axis," Biomedicine \& Pharmacotherapy, vol. 123, p. 109650, 2020.

[37] Z. Chen, J. Zhang, Z. Zhang et al., "The putative tumor suppressor microRNA-30a-5p modulates clear cell renal cell carcinoma aggressiveness through repression of ZEB2," Cell Death \& Disease, vol. 8, no. 6, article e2859, 2017.

[38] F. F. Shen, Y. Pan, H. J. Yang et al., "Decreased expression of SPINT1-AS1 and SPINT1 mRNA might be independent unfavorable prognostic indicators in esophageal squamous cell carcinoma," Oncotargets and Therapy, vol. 12, pp. 47554763, 2019.

[39] C. Li, W. Li, Y. Zhang et al., "Increased expression of antisense lncRNA SPINT1-AS1 predicts a poor prognosis in colorectal cancer and is negatively correlated with its sense transcript," Oncotargets and Therapy, vol. 11, pp. 3969-3978, 2018.

[40] Z. Xiang, S. Song, Z. Zhu et al., "LncRNAs GIHCG and SPINT1-AS1 are crucial factors for pan-cancer cells sensitivity to lapatinib," Frontiers in Genetics, vol. 10, 2019.

[41] Y. Shen, Y. Feng, H. Chen et al., "Focusing on long non-coding RNA dysregulation in newly diagnosed multiple myeloma," Life Sciences, vol. 196, pp. 133-142, 2018.

[42] M. Pljesa-Ercegovac, A. Savic-Radojevic, V. Coric, T. Radic, and T. Simic, "Glutathione transferase genotypes may serve as determinants of risk and prognosis in renal cell carcinoma," BioFactors, vol. 46, no. 2, pp. 229-238, 2020.

[43] V. Sosa, T. Moliné, R. Somoza, R. Paciucci, H. Kondoh, and M. E. LLeonart, "Oxidative stress and cancer: an overview," Ageing Research Reviews, vol. 12, no. 1, pp. 376-390, 2013.

[44] M. Pljesa-Ercegovac, J. Mimic-Oka, D. Dragicevic et al., "Altered antioxidant capacity in human renal cell carcinoma: role of glutathione associated enzymes," Urologic Oncology: Seminars and Original Investigations, vol. 26, no. 2, pp. 175181, 2008.

[45] M. S. Leisegang, K. Schröder, and R. P. Brandes, "Redox regulation and noncoding RNAs," Antioxidants and Redox Signaling, vol. 29, no. 9, pp. 793-812, 2018.

[46] L. Chen, H. Yang, Z. Yi et al., "LncRNA GAS5 regulates redox balance and dysregulates the cell cycle and apoptosis in malignant melanoma cells," Journal of Cancer Research and Clinical Oncology, vol. 145, no. 3, pp. 637-652, 2019.

[47] W. He, B. Liang, C. Wang et al., "MSC-regulated lncRNA MACC1-AS1 promotes stemness and chemoresistance through fatty acid oxidation in gastric cancer," Oncogene, vol. 38 , no. 23, pp. 4637-4654, 2019. 